\title{
NUMERICAL CALCULATION OF THREE-DIMENSIONAL INTERFACIAL POTENTIAL FLOWS USING THE POINT VORTEX METHOD*
}

\author{
DAVID J. HAROLDSEN ${ }^{\dagger}$ AND DANIEL I. MEIRON ${ }^{\ddagger}$
}

\begin{abstract}
An application of the point vortex method to the singular Biot-Savart integrals used in water wave calculations is presented. The error for this approximation is shown to be a series in odd powers of $h$. A method for calculating the coefficients in the series is presented.
\end{abstract}

Key words. point vortex method, vortex sheet, interfacial potential flows

AMS subject classifications. 76B15, 65M99

PII. S1064827596302060

1. Introduction. In a study of vortex methods for free-surface flow problems (in two dimensions), Baker, Meiron, and Orszag [7] applied iterative boundary integral methods which offered advantages both in computational storage and in arithmetic operations when compared with conventional methods which require matrix inversion. Specifically, the authors used boundary integrals to define the interfacial velocity and velocity potential.

In the case of three-dimensional potential flows, the integral which defines the velocity is a singular Biot-Savart integral of the following form for a vortex sheet:

$$
\mathbf{u}(\mathbf{x})=\frac{1}{4 \pi} \int_{S^{\prime}} \boldsymbol{\kappa}\left(\mathbf{x}^{\prime}\right) \times \nabla_{\mathbf{x}} G\left(\mathbf{x}, \mathbf{x}^{\prime}\right) d S^{\prime},
$$

where $\boldsymbol{\kappa}=\hat{\mathbf{n}} \times\left[\mathbf{u}_{1}-\mathbf{u}_{2}\right]$ is the vortex sheet strength, $\hat{\mathbf{n}}$ is the unit normal to the interface, $\mathbf{u}_{1}-\mathbf{u}_{2}$ is the jump in interfacial velocity at the vortex sheet ( $\mathbf{u}_{1}$ is the velocity above the interface), and

$$
G\left(\mathbf{x}, \mathbf{x}^{\prime}\right)=-\frac{1}{\left|\mathbf{x}-\mathbf{x}^{\prime}\right|}
$$

One approach to numerically evaluating this singular integral is the point vortex method. The point vortex method as we and others have applied it is the trapezoidal rule for integration, excluding the singular contribution. Goodman, Hou, and Lowengrub [1] showed that for the Euler equations in two dimensions, the point vortex approximation to the Biot-Savart integral converges and the error is a series in powers of $h^{2}$, where $h$ is the local mesh size. Lowengrub, Shelley, and Merriman [2] explicitly computed the coefficients of the error series for the point vortex approximation to the two dimensional Biot-Savart integral for a rectangular grid. In their study, the authors applied the Poisson summation formula generalized to singular functions to determine the coefficients of the series. Hou and Lowengrub [3]

${ }^{*}$ Received by the editors April 15, 1996; accepted for publication (in revised form) April 7, 1997; published electronically September 10, 1998. This work was supported by NSF grant DMS-9407050. http://www.siam.org/journals/sisc/20-2/30206.html

${ }^{\dagger}$ Department of Mathematical Sciences, U.S. Military Academy, West Point, NY 10996 (ad1256@ exmail.usma.edu). The work of this author was supported in part by a National Science Foundation fellowship.

‡Applied Mathematics, 217-50, California Institute of Technology, Pasadena, CA 91125 (dim@ama.caltech.edu). 


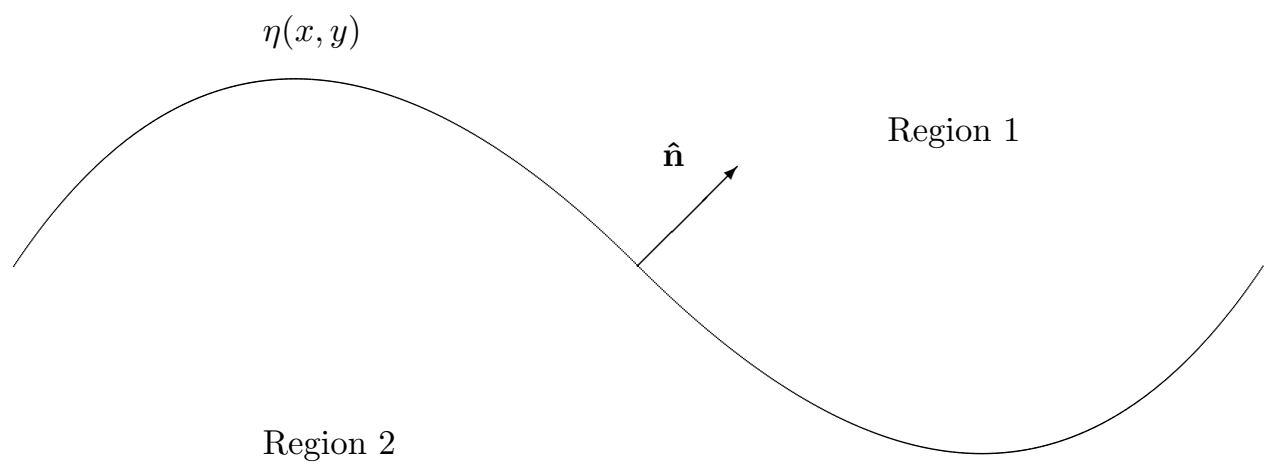

FIG. 2.1. Regions 1 and 2 with the normal vector $\hat{\mathbf{n}}$ pointed into region 1.

also showed convergence of the point vortex method for the Biot-Savart law in the case of the three-dimensional Euler equations. In the case of a vortex sheet in two dimensions, Shelley [9] showed that removing the first-order error term from the point vortex method gives a spectrally accurate method. Hou, Lowengrub, and Krasny [6] proved convergence of the point vortex method using the desingularization technique that Shelley [9] presented.

With these studies in mind, we present an examination of the point vortex method as applied to the case of three-dimensional water waves. This type of nonlinear system is characteristic of interfacial potential flows, and we use it here as an example of our approach. We limit ourselves to the case of waves that are smooth and periodic in $x$ and $y$ and where the wave height is a single-valued function of $x$ and $y$. This latter restriction is not, however, crucial. In this study, we discuss the numerical computation of the singular Biot-Savart integrals, including some practical aspects of implementing their computation. We analyze the error associated with the approximation and discuss the form of the error expansion. Specifically, we show that the error expansion can be represented as a series in odd powers of a discretization parameter $h$. Finally, we demonstrate how to calculate the coefficients of the error expansion and we provide several examples.

2. The singular Biot-Savart integrals. We consider an interface separating two incompressible, inviscid fluids as in Figure 2.1. We define $\eta(x, y, t)$ to be the interfacial height. We label the region above the interface as region 1 and that below as region 2 . The velocity field $\mathbf{u}_{1}\left(\mathbf{u}_{2}\right)$ is the velocity above (below) the interface. We introduce the following definitions of $\mathbf{u}_{+}$and $\mathbf{u}_{-}$:

$$
\begin{aligned}
& \mathbf{u}_{+}=\lim _{z \rightarrow \eta^{+}} \mathbf{u}_{1}, \\
& \mathbf{u}_{-}=\lim _{z \rightarrow \eta^{-}} \mathbf{u}_{2} .
\end{aligned}
$$

The flow in each region is irrotational, so we can introduce the velocity potentials $\phi_{1}$ and $\phi_{2}$ given by $\mathbf{u}_{1}=\nabla \phi_{1}$ and $\mathbf{u}_{2}=\nabla \phi_{2}$. Since the flows are incompressible, the 
velocity potentials satisfy Laplace's equation:

$$
\nabla^{2} \phi_{2}=\nabla^{2} \phi_{1}=0
$$

The kinematic condition that the interface moves with the fluid is given by

$$
\frac{\partial \eta}{\partial t}=w_{-}-u_{-} \frac{\partial \eta}{\partial x}-v_{-} \frac{\partial \eta}{\partial y}
$$

In order to evolve $\eta$ forward in time, we must be able to compute $\mathbf{u}_{-}$, and hence $\phi_{-}$. For this we make use of the Bernoulli equation which expresses the continuity of normal stresses:

$$
\frac{\partial \phi_{2}}{\partial t}+\frac{1}{2}\left(u_{2}^{2}+v_{2}^{2}+w_{2}^{2}\right)+g z=\left.0\right|_{z=\eta(x, y, t)},
$$

For fluid particles on the interface, we have some flexibility in choosing the particle velocity, and we constrain the surface particles to move only in the vertical direction. If we define the vertical particle velocity to be $w$, then the modified Bernoulli equation is

$$
\frac{D \phi_{2}}{D t}-w w_{2}+\frac{1}{2}\left(u_{2}^{2}+v_{2}^{2}+w_{2}^{2}\right)+g z=0
$$

At $z=\eta$, this equation becomes

$$
\frac{D \phi_{-}}{D t}-w w_{-}+\frac{1}{2}\left(u_{-}^{2}+v_{-}^{2}+w_{-}^{2}\right)+g \eta=0 .
$$

Equations (2.4) and (2.7) define the time evolution of $\eta$ and $\phi_{-}$respectively. In order to use these evolution equations, we need a method for computing the velocities. The method we develop here relies on singular Biot-Savart integrals.

The interface can be considered as a distribution of dipoles of strength density $\hat{\mathbf{n}}\left(\phi_{-}-\phi_{+}\right) / 4 \pi$ (see, for example, Saffman [10]). The potential at a point off the interface is defined as

$$
\phi(x, y)=-\frac{1}{4 \pi} \int_{S^{\prime}} \mu\left(\mathbf{x}^{\prime}\right) \hat{\mathbf{n}}\left(\mathbf{x}^{\prime}\right) \cdot \nabla^{\prime} G\left(\mathbf{x}, \mathbf{x}^{\prime}\right) d S^{\prime},
$$

where $\hat{\mathbf{n}}$ is the normal to the surface, $\mu=\phi_{-}-\phi_{+}$, and

$$
G\left(\mathbf{x}, \mathbf{x}^{\prime}\right)=-\frac{1}{\left|\mathbf{x}-\mathbf{x}^{\prime}\right|} \text {. }
$$

Depending on the direction from which we approach the interface, the potential assumes the value $\phi_{-}$or $\phi_{+}$. On the interface, the integral is defined by a principal value integral, which we denote by $\phi$. The value $\phi$ is the mean of the two limits:

$$
\phi=\frac{\phi_{+}+\phi_{-}}{2} .
$$

Using this relation and the definition of $\mu$, we obtain a Fredholm integral equation of the second kind:

$$
\mu=2 \phi_{-}-2 \phi=2 \phi_{-}+\frac{1}{2 \pi} \int_{S^{\prime}} \mu\left(\mathbf{x}^{\prime}\right) \hat{\mathbf{n}} \cdot \nabla^{\prime} G\left(\mathbf{x}, \mathbf{x}^{\prime}\right) d S^{\prime},
$$


where the integral is a principal value integral. This equation can be solved by iteration on $\mu$. The fluid velocity off the interface can also be defined using a boundary integral (see Saffman [10]):

$$
\mathbf{u}(x, y, z)=\frac{1}{4 \pi} \int_{S^{\prime}} \kappa\left(\mathbf{x}^{\prime}\right) \times \nabla_{\mathbf{x}} G\left(\mathbf{x}, \mathbf{x}^{\prime}\right) d S^{\prime},
$$

where the vortex sheet strength $\boldsymbol{\kappa}$ is defined by

$$
\boldsymbol{\kappa}(x, y)=\hat{\mathbf{n}} \times\left(\mathbf{u}_{+}-\mathbf{u}_{-}\right) .
$$

We define $\mathbf{u}_{+}$and $\mathbf{u}_{-}$as the limit of $\mathbf{u}$ as $z$ approaches $\eta$ from above and below, respectively. The fluid velocity on the interface $\mathbf{u}(x, y)$ is defined by a principal value integral whose value is given by

$$
\mathbf{u}(x, y)=\frac{\mathbf{u}_{+}+\mathbf{u}_{-}}{2} .
$$

If we know $\mu$, we can calculate the vortex sheet strength $\boldsymbol{\kappa}$. To do this, we define $\mathbf{r}=x \hat{\mathbf{i}}+y \hat{\mathbf{j}}+\eta(x, y) \hat{\mathbf{k}}$ to represent a point on the interface. We note that

$$
\phi_{-}(x, y)=\phi_{1}(x, y, \eta(x, y))
$$

and take the derivative of $\phi_{-}$with respect to $x$. Doing this (and making use of the chain rule) gives

$$
\begin{aligned}
\frac{\partial}{\partial x} \phi_{-}(x, y) & =\frac{\partial}{\partial x} \phi_{1}(x, y, \eta(x, y)) \\
& =u_{1}(x, y, \eta(x, y))+\frac{\partial \eta}{\partial x} w_{1}(x, y, \eta(x, y)) \\
& =u_{-}(x, y)+\frac{\partial \eta}{\partial y} w_{-}(x, y) \\
& =\frac{\partial \mathbf{r}}{\partial x} \cdot \mathbf{u}_{-} .
\end{aligned}
$$

We can similarly derive the following:

$$
\frac{\partial \phi_{-}}{\partial y}=\frac{\partial \mathbf{r}}{\partial y} \cdot \mathbf{u}_{-} .
$$

Similar relations involving $\phi_{+}$and $\mathbf{u}_{+}$can also be derived. Using these relations together with the requirement that the normal velocity at the interface be continuous, we can derive the following relation:

$$
\mathbf{u}_{-}-\mathbf{u}_{+}=\frac{\left(\frac{\partial \mathbf{r}}{\partial y} \times \hat{\mathbf{n}}\right) \frac{\partial \mu}{\partial x}-\left(\frac{\partial \mathbf{r}}{\partial x} \times \hat{\mathbf{n}}\right) \frac{\partial \mu}{\partial y}}{\left|\frac{\partial \mathbf{r}}{\partial x} \times \frac{\partial \mathbf{r}}{\partial y}\right|}
$$

If we define

$$
\gamma_{x}(x, y)=\frac{\partial}{\partial x} \mu(x, y) \text { and } \gamma_{y}(x, y)=\frac{\partial}{\partial y} \mu(x, y)
$$


then we have the following expression for $\kappa$ in terms of $\mu$ :

$$
\boldsymbol{\kappa}(x, y)=\hat{\mathbf{n}} \times\left[\mathbf{u}_{+}-\mathbf{u}_{-}\right]=\frac{\gamma_{y} \hat{\mathbf{\imath}}-\gamma_{x} \hat{\mathbf{j}}+\left(\frac{\partial \eta}{\partial x} \gamma_{y}-\frac{\partial \eta}{\partial y} \gamma_{x}\right) \hat{\mathbf{k}}}{\sqrt{1+\left(\frac{\partial \eta}{\partial x}\right)^{2}+\left(\frac{\partial \eta}{\partial y}\right)^{2}}} .
$$

If we know $\mathbf{u}=(u, v, w)$, we can compute $\mathbf{u}_{-}$as follows:

$$
\begin{aligned}
& w_{-}=\frac{w-\frac{\partial \eta}{\partial x}\left(u-\frac{\partial \phi_{-}}{\partial x}\right)-\frac{\partial \eta}{\partial y}\left(v-\frac{\partial \phi_{-}}{\partial y}\right)}{1+\left(\frac{\partial \eta}{\partial x}\right)^{2}+\left(\frac{\partial \eta}{\partial y}\right)^{2}}, \\
& u_{-}=\frac{\partial \phi_{-}}{\partial x}-w_{-} \frac{\partial \eta}{\partial x} \\
& v_{-}=\frac{\partial \phi_{-}}{\partial y}-w_{-} \frac{\partial \eta}{\partial y} .
\end{aligned}
$$

Thus, if we initially know $\eta(x, y)$ and $\phi_{-}(x, y)$, we can step the evolution equations (2.4) and (2.7) forward in time as follows:

1. Compute $\mu$ from the Fredholm integral equation (2.11).

2. Compute $\boldsymbol{\kappa}$ from $\mu(2.20)$.

3. Compute $\mathbf{u}=(u, v, w)$ from $\boldsymbol{\kappa}(2.12)$.

4. Compute $\mathbf{u}_{-}=\left(u_{-}, v_{-}, w_{-}\right)$from $\mathbf{u}(2.21)$.

5. Step $\eta$ and $\phi_{-}$forward in time (2.4), (2.7).

Having established this algorithm, it is easily seen that the most computationally intensive (and difficult) step is step 3, the computation of $\mathbf{u}$ from the Biot-Savart law. (The computation of $\phi$ is also time consuming, but the integral is less singular.) In the following sections, we outline and analyze a method for computing $\mathbf{u}$.

3. The point vortex method. We are interested in the computation of the singular Biot-Savart integrals which define the velocity and the velocity potential. We limit ourselves to the former and note that the analysis of the integral defining the potential is similar. We assume that $\eta, \gamma_{x}$, and $\gamma_{y}$ are periodic in $x$ and $y$ with period $2 \pi$. We can express the velocity integral as follows:

$$
\begin{aligned}
\mathbf{u}(x, y) & =\frac{1}{4 \pi} \int_{S^{\prime}} \boldsymbol{\kappa}\left(\mathbf{x}^{\prime}\right) \times \nabla_{\mathbf{x}} G\left(\mathbf{x}, \mathbf{x}^{\prime}\right) d S^{\prime} \\
& =\frac{1}{4 \pi} \int_{-\infty}^{\infty} \int_{-\infty}^{\infty} \frac{[\alpha \hat{\mathbf{l}}+\beta \hat{\mathbf{j}}+\delta \hat{\mathbf{k}}] d x^{\prime} d y^{\prime}}{\left[\left(x-x^{\prime}\right)^{2}+\left(y-y^{\prime}\right)^{2}+\left(\eta(x, y)-\eta\left(x^{\prime}, y^{\prime}\right)\right)^{2}\right]^{3 / 2}}
\end{aligned}
$$

where

$$
\begin{aligned}
\alpha= & {\left[\frac{\partial \eta}{\partial y}\left(x^{\prime}, y^{\prime}\right) \gamma_{x}\left(x^{\prime}, y^{\prime}\right)-\frac{\partial \eta}{\partial x}\left(x^{\prime}, y^{\prime}\right) \gamma_{y}\left(x^{\prime}, y^{\prime}\right)\right]\left(y-y^{\prime}\right) } \\
& -\gamma_{x}\left(x^{\prime}, y^{\prime}\right)\left[\eta(x, y)-\eta\left(x^{\prime}, y^{\prime}\right)\right], \\
\beta= & {\left[\frac{\partial \eta}{\partial x}\left(x^{\prime}, y^{\prime}\right) \gamma_{y}\left(x^{\prime}, y^{\prime}\right)-\frac{\partial \eta}{\partial y}\left(x^{\prime}, y^{\prime}\right) \gamma_{x}\left(x^{\prime}, y^{\prime}\right)\right]\left(x-x^{\prime}\right) } \\
& -\gamma_{y}\left(x^{\prime}, y^{\prime}\right)\left[\eta(x, y)-\eta\left(x^{\prime}, y^{\prime}\right)\right], \\
\delta= & \gamma_{x}\left(x^{\prime}, y^{\prime}\right)\left(x-x^{\prime}\right)+\gamma_{y}\left(x^{\prime}, y^{\prime}\right)\left(y-y^{\prime}\right) .
\end{aligned}
$$


In order to simplify the notation, consider the evaluation of the velocity at the origin. That is, we set $(x, y)=(0,0)$ and drop the primes. Our conclusions are valid for general $(x, y)$. We also limit discussion to the evaluation of the $z$-component of $\mathbf{u}(x, y)=(u, v, w)$. The cases involving the other components are completely analogous. Applying the simplifications mentioned above gives

$$
w(0,0)=-\frac{1}{4 \pi} \int_{-\infty}^{\infty} \int_{-\infty}^{\infty} \frac{x \gamma_{x}(x, y)+y \gamma_{y}(x, y)}{\left[x^{2}+y^{2}+(\eta(0,0)-\eta(x, y))^{2}\right]^{3 / 2}} d x d y
$$

We can take advantage of the periodicity of $\eta, \gamma_{x}$, and $\gamma_{y}$ to redefine $w(0,0)$ in such a way that the domain of integration is finite. We then have

$$
\begin{aligned}
w(0,0)= & -\frac{1}{4 \pi} \int_{-\pi}^{\pi} \int_{-\pi}^{\pi} d x d y \\
& \times \sum_{n=-\infty}^{\infty} \sum_{m=-\infty}^{\infty} \frac{\gamma_{x}(x, y)(x-2 n \pi)+\gamma_{y}(x, y)(y-2 m \pi)}{\left[(x-2 n \pi)^{2}+(y-2 m \pi)^{2}+(\eta(0,0)-\eta(x, y))^{2}\right]^{3 / 2}} .
\end{aligned}
$$

In order to evaluate this lattice sum, we have applied the Ewald summation technique as outlined by Baker, Meiron, and Orszag [8], which converts the derivatives of the periodic Green's function into sums of error functions.

The point vortex method is an application of the two-dimensional trapezoidal rule, excluding the singular contribution. If we define the integrand to be $f(x, y)$ and denote the approximation of $w$ by $w_{A}(0,0)$, then the method is defined as follows:

$$
w_{A}(0,0)=-\frac{1}{4 \pi} h^{2} \sum_{i=-n / 2+1}^{n / 2} \sum_{\substack{j=-n / 2+1 \\(i, j) \neq 0}}^{n / 2} f(i h, j h),
$$

where $h=2 \pi / n$.

We are interested in the error

$$
E=w_{A}(0,0)-w(0,0) .
$$

Based on the studies mentioned in the introduction, we suspect that $E$ should be a series in powers of $h$. If this is the case, then we can use Richardson extrapolation to obtain a higher-order method for computing $w$. In practice, we apply Richardson extrapolation by noting that it is equivalent to assigning weights to each grid point. This gives a modified definition of $w_{A}$ :

$$
w_{A}(0,0)=-\frac{1}{4 \pi} h^{2} \sum_{i=-n / 2+1}^{n / 2} \sum_{\substack{j=-n / 2+1 \\(i, j) \neq 0}}^{n / 2} w_{i j} f(i h, j h) .
$$

Here $w_{i j}$ is the weight associated with Richardson extrapolation. As an example, let $I_{A}[h]$ be the numerical approximation to $I$. If we know that

$$
I_{A}[h]=I+C_{1} h+C_{2} h^{2}+\cdots,
$$

then we can use Richardson extrapolation by noting that

$$
I_{A}[2 h]=I+2 C_{1} h+4 C_{2} h^{2}+\cdots .
$$


Subtracting $I_{A}[2 h]$ from $2 I_{A}[h]$ gives

$$
2 I_{A}[h]-I_{A}[2 h]=I-2 C_{2} h^{2}+\cdots .
$$

We see that the first order term has been removed, leaving a second-order approximation for $I$. To determine the weights $w_{i j}$, we define the function $g(i, j)$ to be

$$
g(i, j)= \begin{cases}1 & \text { if } i \text { and } j \text { are both even } \\ 0 & \text { otherwise }\end{cases}
$$

Using this definition, we write

$$
\begin{aligned}
2 I_{A}[h]-I_{A}[2 h]=-\frac{2}{4 \pi} h^{2} \sum_{i=-n / 2+1}^{n / 2} \sum_{\substack{j=-n / 2+1 \\
(i, j) \neq 0}}^{n / 2} f(i h, j h) \\
+\frac{1}{4 \pi} 4 h^{2} \sum_{\substack { i=-n / 4+1 \\
\begin{subarray}{c}{j=-n / 4+1 \\
(i, j) \neq 0{ i = - n / 4 + 1 \\
\begin{subarray} { c } { j = - n / 4 + 1 \\
( i , j ) \neq 0 } }\end{subarray}}^{n / 4} f(2 i h, 2 j h) \\
=-\frac{1}{4 \pi} h^{2} \sum_{i=-n / 2+1}^{n / 2} \sum_{\substack{j=-n / 2+1 \\
(i, j) \neq 0}}^{n / 2}[2-4 g(i, j)] f(i h, j h) .
\end{aligned}
$$

From this we see that the definition of $w_{i j}$ for a second-order method would be

$$
w_{i j}=2-4 g(i, j) \text {. }
$$

The condition that $g(i, j)=1$ occurs when $i$ and $j$ are both even, and this occurs when the lattice point $(i, j)$ contributes to both $I_{A}[h]$ and $I_{A}[2 h]$. Thus, lattice points contributing only to $I_{A}[h]$ have $w_{i j}=2$ while those contributing to both sums have $w_{i j}=-2$.

In our numerical experiments with (3.6), the application of Richardson extrapolation led us to believe that $E$ can be expressed as a series in odd powers of $h$. In Figure 3.1, we show a calculation of the error associated with the point-vortex method and the first two applications of Richardson extrapolation. In Figure 3.2 we show the slopes (in absolute value) of the respective error curves in Figure 3.1. In this example, we have taken

$$
\begin{aligned}
\eta & =\sin (x+y)+\cos ^{2}(x+y), \\
\gamma_{x} & =0 \\
\gamma_{y} & =-2[\sin (y)+\cos (y)] .
\end{aligned}
$$

The figures show (as expected) that the point vortex method is a first-order method. The successive application of Richardson extrapolation yields third- and fifth-order methods, respectively. This suggests that the error is a series in odd powers of $h$. The analysis of the method in the next section confirms this claim.

4. Analysis of the point vortex method. In order to justify the general use of Richardson extrapolation, we must demonstrate that the error associated with the point vortex method has the appropriate form. Our analysis of the method relies on two different approaches. The first approach, which demonstrates that the error is a 


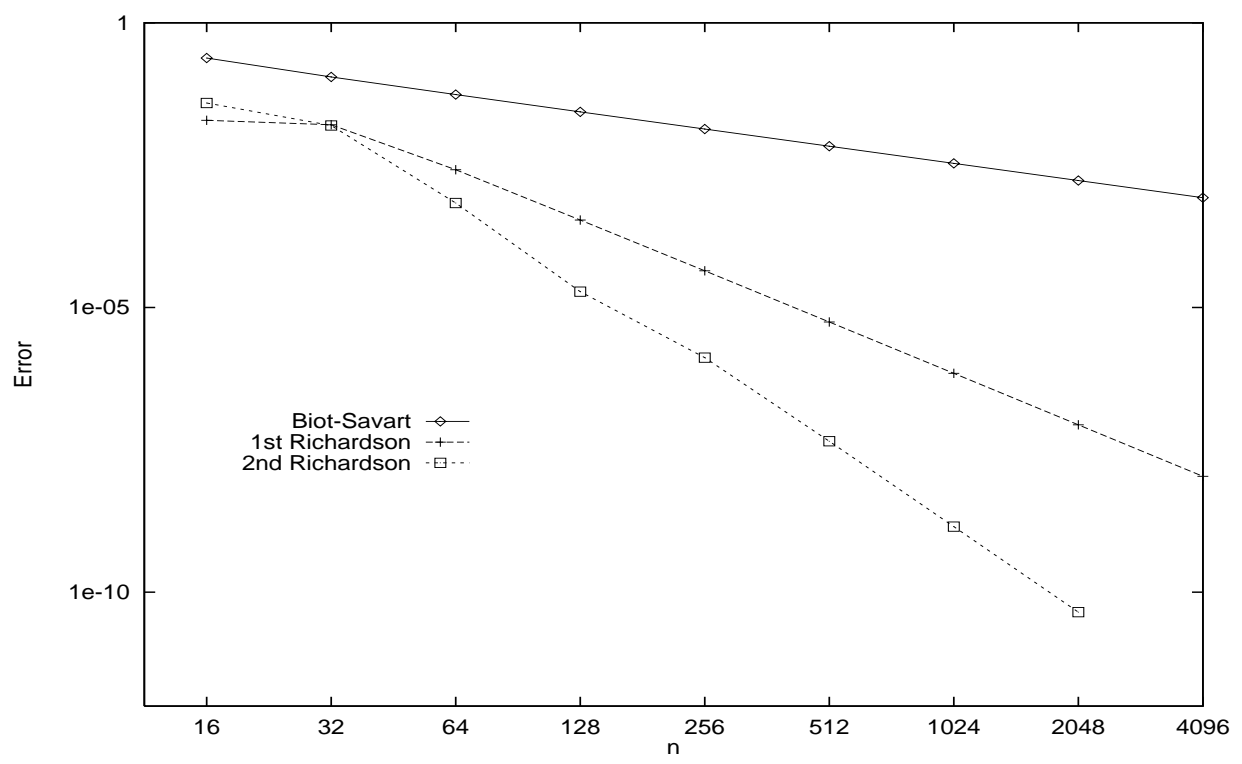

FIG. 3.1. The line labeled Biot-Savart gives the error from the point vortex method. The other lines give the error for the first two applications of Richardson extrapolation.

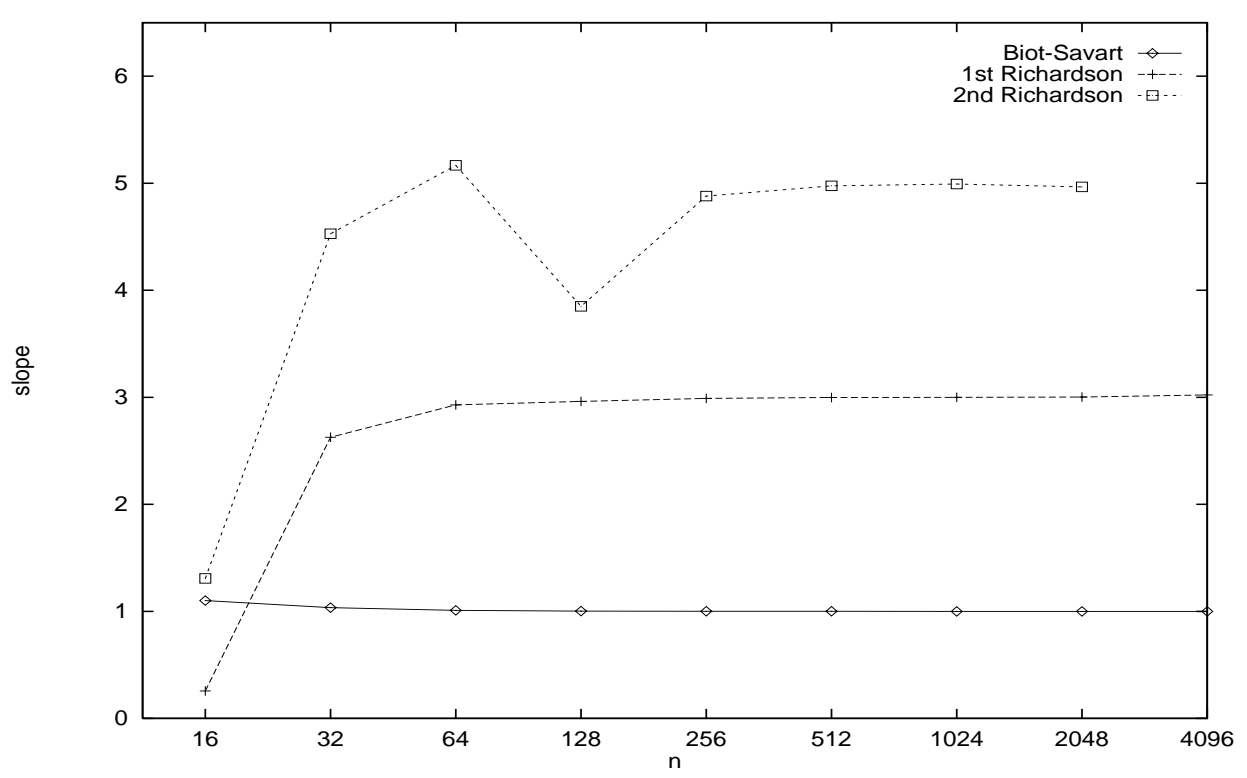

FIG. 3.2. The order of the three different methods demonstrated in Figure 3.1.

series in odd powers of $h$, is based on work by Goodman, Hou, and Lowengrub [1]. The second method is an application of the two-dimensional Euler-Maclaurin summation formula which demonstrates how to construct the coefficients in this series. Because of the comparative simplicity of the method of Goodman, Hou, and Lowengrub [1], we discuss their ideas first. 
4.1. The form of the error. We consider the point vortex approximation to the integral

$$
I=\int_{-\infty}^{\infty} \int_{-\infty}^{\infty} K(x, y) p(x, y) d x d y
$$

where

$$
K(x, y)=\frac{1}{\left[x^{2}+y^{2}+(\eta(0,0)-\eta(x, y))^{2}\right]^{3 / 2}}
$$

and

$$
p(x, y)=-\frac{1}{4 \pi}\left[x \gamma_{x}(x, y)+y \gamma_{y}(x, y)\right] .
$$

We assume that $\gamma_{x}$ and $\gamma_{y}$ are smooth, periodic, and bounded in $x$ and $y$. The error $E$ associated with this approximation is defined by

$$
E=h^{2} \sum_{i=-\infty}^{\infty} \sum_{\substack{j=-\infty \\(i, j) \neq(0,0)}}^{\infty} K\left(x_{i}, y_{j}\right) p\left(x_{i}, y_{j}\right)-\int_{-\infty}^{\infty} \int_{-\infty}^{\infty} K(x, y) p(x, y) d x d y,
$$

where we use the notation $\left(x_{i}, y_{j}\right)=(i h, j h)$.

Following the treatment of Goodman, Hou, and Lowengrub [1], we split $E$ into a near-field part, $\sigma$, and a far-field part, $\tau$. To do this, we define a cutoff function $f_{1}(s)$. We take $f_{1}$ to be a function which is smooth for all $s$ and which satisfies the following: $f_{1}(s) \equiv 1$ if $|s| \leq 1, f_{1}(s) \equiv 0$ if $|s| \geq 2$, and $f_{1}(s)$ varies smoothly from 0 to 1 for $1 \leq|s| \leq 2$. We also define $f_{2}(s)=1-f_{1}(s)$. We take $0<q<1$ and define

$$
\begin{aligned}
\sigma= & h^{2} \sum_{i=-\infty}^{\infty} \sum_{\substack{j=-\infty \\
(i, j) \neq 0}}^{\infty} K\left(x_{i}, y_{j}\right) p\left(x_{i}, y_{j}\right) f_{1}\left(h^{-q}|\mathbf{x}|\right) \\
& -\int_{-\infty}^{\infty} \int_{-\infty}^{\infty} K(x, y) p(x, y) f_{1}\left(h^{-q}|\mathbf{x}|\right) d x d y
\end{aligned}
$$

and

$$
\begin{aligned}
\tau= & h^{2} \sum_{i=-\infty}^{\infty} \sum_{\substack{j=-\infty \\
(i, j) \neq 0}}^{\infty} K\left(x_{i}, y_{j}\right) p\left(x_{i}, y_{j}\right) f_{2}\left(h^{-q}\left|\mathbf{x}_{\mathbf{k}}\right|\right) d x d y \\
& -\int_{-\infty}^{\infty} \int_{-\infty}^{\infty} K(x, y) p(x, y) f_{2}\left(h^{-q}|\mathbf{x}|\right) d x d y,
\end{aligned}
$$

where $\mathbf{x}=(x, y)$ and $\mathbf{x}_{\mathbf{k}}=\left(x_{i}, y_{j}\right)$. To analyze $\sigma$, we express $K(x, y) p(x, y)$ in a series expansion in $x$ and $y$. Since $p(x, y)$ has no singularities, we have the following expansion for $p$ :

$$
p(x, y)=p_{x}^{0} x+p_{y}^{0} y+\cdots+O\left(|\mathbf{x}|^{N}\right)
$$

for any $N$, where we use the notation

$$
p_{x}^{0}=\left.\frac{\partial}{\partial x} p\right|_{(x, y)=(0,0)} .
$$


We expand the denominator of $K(x, y)$ as follows:

$$
\begin{aligned}
& {\left[x^{2}+y^{2}+(\eta(0,0)-\eta(x, y))^{2}\right]^{3 / 2}} \\
& =x^{2}+y^{2}+\left(\eta_{x}^{0} x+\eta_{y}^{0} y\right)^{2}+\left(\eta_{x}^{0} x+\eta_{y}^{0} y\right)\left(\eta_{x x}^{0} x^{2}+\eta_{y y}^{0}+2 \eta_{x y}^{0} x y\right)+\cdots+O\left(|\mathbf{x}|^{N}\right) \\
& =\left(a x^{2}+b y^{2}+d x y\right)^{3 / 2}\left(1+\frac{\left(\eta_{x}^{0} x+\eta_{y}^{0} y\right)\left(\eta_{x x}^{0} x^{2}+\eta_{y y}^{0}+2 \eta_{x y}^{0} x y\right)}{\left(a x^{2}+b y^{2}+d x y\right)^{3 / 2}}+\cdots\right)^{3 / 2}
\end{aligned}
$$

for any $N$. In this equation, we have

$$
\begin{aligned}
& a=1+\eta_{x}^{2}(0,0), \\
& b=1+\eta_{y}^{2}(0,0), \\
& d=2 \eta_{x}(0,0) \eta_{y}(0,0) .
\end{aligned}
$$

From the above results we have that

$$
K(x, y) p(x, y)=C_{0}(x, y)+C_{1}(x, y)+C_{2}(x, y)+\cdots+O\left(|\mathbf{x}|^{N}\right), .
$$

where $C_{n}(x, y)$ is homogeneous of degree $n-2$ in $\mathbf{x}$. In addition, $C_{n}$ is even in $\mathbf{x}$ for odd $n$ and odd in $\mathbf{x}$ for even $n$. Since $f_{1}(|\mathbf{x}|)$ is even in $\mathbf{x}$, we have

$$
\int_{-\infty}^{\infty} \int_{-\infty}^{\infty} C_{n}(x, y) f_{1}\left(h^{-q}|\mathbf{x}|\right) d x d y=0
$$

for $n$ even. The substitution $(x, y)=h^{q}\left(\eta_{1}, \eta_{2}\right)$ gives, for $n$ odd, that

$$
\int_{-\infty}^{\infty} \int_{-\infty}^{\infty} C_{n}(x, y) f_{1}\left(h^{-q}|\mathbf{x}|\right) d x d y=h^{q n} \int_{-\infty}^{\infty} \int_{-\infty}^{\infty} C_{n}\left(\eta_{1}, \eta_{2}\right) f(|\eta|) d \eta_{1} d \eta_{2}
$$

where $\eta=\left(\eta_{1}, \eta_{2}\right)$. We define $a_{n}$ as follows:

$$
a_{n}=\int_{-\infty}^{\infty} \int_{-\infty}^{\infty} C_{n}\left(\eta_{1}, \eta_{2}\right) f(|\eta|) d \eta_{1} d \eta_{2}
$$

We now analyze the discrete sum in $\sigma$ (4.5). Again using the fact that $f_{1}(\mathbf{x})$ is even in $\mathbf{x}$ and $C_{n}(x, y)$ is odd in $\mathbf{x}$ for odd $n$, we have

$$
h^{2} \sum_{i=-\infty}^{\infty} \sum_{\substack{j=-\infty \\(i, j) \neq(0,0)}}^{\infty} C_{n}(h i, h j) f_{1}\left(h^{1-q}|k|\right)=0
$$

for $n$ even, where we define $k=(i, j)$. For $n$ odd, we make use of the homogeneity of $C_{n}$ to rewrite the sum as follows:

$$
h^{2} \sum_{i=-\infty}^{\infty} \sum_{\substack{j=-\infty \\(i, j) \neq(0,0)}}^{\infty} C_{n}(h i, h j) f_{1}\left(h^{1-q}|k|\right)=h^{n} S_{n}(h),
$$

where

$$
S_{n}(h)=\sum_{i=-\infty}^{\infty} \sum_{\substack{j=-\infty \\(i, j) \neq(0,0)}}^{\infty} C_{n}(i, j) f_{1}\left(h^{1-q}|k|\right) .
$$


Goodman, Hou, and Lowengrub [1] show that $S_{n}(h)$ can be expressed in the following form:

$$
S_{n}(h)=D_{n}+b_{n} h^{-n(1-q)}+O\left(h^{N}\right) \text { for any } N,
$$

where $D_{n}$ is constant and $b_{n}$ depends on the cutoff function $f_{1}$. Combining this result with the result for the integral terms (4.16) gives

$$
\sigma=D_{1} h+D_{3} h^{3}+\cdots+d_{1} h^{q}+d_{3} h^{3 q}+\cdots+O\left(h^{N}\right) \text { for any } N,
$$

where we take $d_{i}=b_{i}-a_{i}$.

The theory of numerical quadrature tells us that $\tau=o\left(h^{N}\right)$ for any $N$ since the integrand has no singularities and decays at infinity. This gives

$$
E=\sigma+\tau=D_{1} h+D_{3} h^{3}+\cdots+d_{1} h^{q}+d_{3} h^{3 q}+\cdots+O\left(h^{N}\right) .
$$

Since $E$ must be independent of $q$, we conclude that $d_{i}=0$ for any $i$. This leaves

$$
E=\sigma+\tau=D_{1} h+D_{3} h^{3}+\cdots+O\left(h^{N}\right) .
$$

The fact that we have a series in odd powers of $h$ rather than in even powers of $h$ (as in Goodman, Hou, and Lowengrub [1]) is due to the form of $K$.

4.2. Computation of the coefficients. The computation of the coefficients is based on studies of the Euler-Maclaurin summation formula. Our approach follows closely that of Sidi and Israeli [5] and Navot [4], who studied one-dimensional singular integrals. We first sketch the approach and then cite some examples.

Recall that we are studying integrals of the form

$$
I=\int_{-\infty}^{\infty} \int_{-\infty}^{\infty} K(x, y) p(x, y) d x d y
$$

where $K(x, y)$ is defined by $(4.2)$ and

$$
\begin{aligned}
p(x, y) & =-\frac{x \gamma_{x}(x, y)+y \gamma_{y}(x, y)}{4 \pi} \\
& =x g(x, y)+y q(x, y) .
\end{aligned}
$$

As before, we assume that $\gamma_{x}(x, y)$ and $\gamma_{y}(x, y)$ are smooth and periodic in $x$ and $y$, with period $2 \pi$ in each variable. We take $I_{A}$ to be the point-vortex approximation to $I$ :

$$
I_{A}=h^{2} \sum_{i=-\infty}^{\infty} \sum_{\substack{j=-\infty \\(i, j) \neq 0}}^{\infty} f\left(x_{i}, y_{j}\right),
$$

where we have defined

$$
f(x, y)=K(x, y) p(x, y) .
$$

The error $E$ is defined by $E=I_{A}-I$.

The derivation of the coefficients follows the method outlined by Navot [4], which extended the (one-dimensional) Euler-Maclaurin summation formula to include functions with branch singularities. Navot's basic approach is to apply the Euler-Maclaurin 
formula to a region where the integrand is nonsingular and treat the singular region separately. We here outline the approach and leave the details to the appendix. We begin by defining the following:

$$
\begin{gathered}
T=\mathbb{R}^{2} /\{[-h, h] \times[-h, h]\}, \\
I_{T}=\iint_{T} f(x, y) d x d y, \\
I_{S}=\int_{-h}^{h} \int_{-h}^{h} f(x, y) d x d y, \\
I_{A_{S}}=\frac{f(h, h)+f(h,-h)+f(-h, h)+f(-h,-h)}{4} \\
+\frac{f(0, h)+f(h, 0)+f(0,-h)+f(-h, 0)}{2} .
\end{gathered}
$$

We rewrite $I$ as follows:

$$
\begin{aligned}
I & =\int_{-\infty}^{\infty} \int_{-\infty}^{\infty} f(x, y) d x d y \\
& =I_{T}+I_{S} .
\end{aligned}
$$

We define $I_{A_{T}}$ as follows:

$$
I_{A_{T}}=I_{A}-I_{A_{S}} .
$$

We have defined $I_{A_{S}}$ in such a way that $I_{A_{T}}$ is the trapezoidal rule approximation to $I_{T}$. We then use the Euler-Maclaurin formula to analyze $E_{T}$ :

$$
E_{T}=I_{A_{T}}-I_{T}
$$

We analyze $E_{S}=I_{A_{S}}-I_{S}$ by expanding $p(x, y)$ in a series about $(0,0)$. Combining the results for $E_{T}$ and $E_{S}$ yields a series in odd powers of $h$. We remark that we have subdivided the domain of integration in such a way that the Euler-Maclaurin formula is applied only on a domain where the integrand and its derivatives are nonsingular.

The Euler-Maclaurin formula is usually applied to integrals where the domain of integration is independent of $h$. Our application of the Euler-Maclaurin formula is different in that we apply the formula to an integral where the domain of integration depends on $h$. We note that while the domain upon which the formula is applied is arbitrarily close to the singular point, the use of the formula is valid since the integrand and its derivatives are nonsingular on the domain. We also note that this approach can be used to study the error in approximating each of the singular integrals that we compute using the point vortex method.

4.3. Examples. In order to simplify the notation, we make the following definitions:

$$
D_{x}^{j} f \equiv \frac{\partial^{j} f}{\partial x^{j}}, \quad \hat{D}_{x}^{j} f \equiv P_{j}(x) \frac{\partial^{j} f}{\partial x^{j}} .
$$

The function $P_{m}(x)$ is given by

$$
P_{m}(x)=\frac{\bar{B}_{m}(x)}{m !}
$$


where $\bar{B}_{m}(x)$ is the Bernoulli polynomial of order $m$ on $[0,1]$ extended periodically. $B_{i}$ is the $i$ th Bernoulli number.

Because the notation and formulas are lengthy and somewhat cumbersome, we present the complete formula only for the simplest case involving a flat interface. We assume the following:

$$
\begin{aligned}
\eta(x, y) & \equiv 0, \\
\gamma_{y}(x, y) & \equiv 0, \\
\gamma_{x}(x, y) & \equiv \gamma_{x}(x) .
\end{aligned}
$$

Given these assumptions, the formula for the error expansion is

$$
E=\sum_{k=1}^{m-1} D_{2 k-1} h^{2 k-1}+O\left(h^{2 m-1}\right),
$$

where

$$
\begin{aligned}
& D_{2 k-1}=-4 \sum_{\gamma=1}^{m} \frac{B_{2 \gamma}}{(2 \gamma) !}\left[\int_{0}^{1} D_{x}^{2 \gamma-1} C_{2 k-1}(1, y) d y+\int_{0}^{1} D_{y}^{2 \gamma-1} C_{2 k-1}(x, 1) d x\right. \\
& +\sum_{\mu=1}^{m} \frac{B_{2 \mu}}{(2 \mu !)} D_{x}^{2 \gamma-1} D_{y}^{2 \mu-1} C_{2 k-1}(1,1) \\
& +\int_{0}^{1} \hat{D}_{x}^{2 m+1} D_{y}^{2 \gamma-1} C_{2 k-1}(x, 1) d x \\
& \left.+\int_{0}^{1} \hat{D}_{x}^{2 \gamma-1} D_{y}^{2 m+1} C_{2 k-1}(1, y) d y\right] \\
& +4 \int_{1}^{\infty} \int_{0}^{\infty} \hat{D}_{x}^{2 m+1} C_{2 k-1}(x, y) d y d x+4 \int_{0}^{1} \int_{1}^{\infty} \hat{D}_{x}^{2 m+1} C_{2 k-1}(x, y) d y d x \\
& +4 \int_{1}^{\infty} \int_{0}^{\infty} \hat{D}_{y}^{2 m+1} C_{2 k-1}(x, y) d x d y+4 \int_{0}^{1} \int_{1}^{\infty} \hat{D}_{y}^{2 m+1} C_{2 k-1}(x, y) d x d y \\
& +4 \int_{1}^{\infty} \int_{0}^{\infty} \hat{D}_{x}^{2 m+1} \hat{D}_{y}^{2 m+1} C_{2 k-1}(x, y) d y d x \\
& +4 \int_{0}^{1} \int_{1}^{\infty} \hat{D}_{x}^{2 m+1} \hat{D}_{y}^{2 m+1} C_{2 k-1}(x, y) d y d x \\
& -4 \int_{0}^{1} \int_{0}^{1} C_{2 k-1}(x, y) d x d y+\left[C_{2 k-1}(1,0)+C_{2 k-1}(1,1)\right]
\end{aligned}
$$

and

$$
C_{k}(x, y)=\frac{\gamma_{x}^{k}(0) x^{k+1}}{k !\left(x^{2}+y^{2}\right)^{3 / 2}}
$$

We note here that given any $m$, we can calculate $D_{2 k-1}$ for all $k$ up to and including $k=m-1$. For example, to calculate $D_{1}$, we need $m \geq 2$. We also observe that (4.41) suggests that there are multiple representations for the coefficients $D_{2 k-1}$. The different representations depend on the value of $m$ chosen. Because $m$ is an arbitrary parameter, the value of the coefficients should be independent of $m$. We have done some numerical experiments which verify this fact. 
We demonstrate the method by calculating $D_{1}$ and $D_{3}$ for a simple problem. We take $\gamma_{x}(x, y)=-2 \sin (x), \gamma_{y} \equiv 0$, and $\eta \equiv 0$. Recalling (4.2) and (4.3), we have

$$
K(x, y) p(x, y)=\frac{x \sin (x)}{2 \pi\left(x^{2}+y^{2}\right)^{3 / 2}}
$$

Expanding $\sin (x)$, we calculate $C_{1}$ and $C_{3}$ as follows:

$$
\begin{aligned}
& C_{1}=\frac{x^{2}}{2 \pi\left(x^{2}+y^{2}\right)^{3 / 2}}, \\
& C_{3}=-\frac{x^{4}}{12 \pi\left(x^{2}+y^{2}\right)^{3 / 2}} .
\end{aligned}
$$

Taking $m=3$, we have expressions for $D_{1}$ and $D_{3}$ from (4.41):

$$
\begin{aligned}
& D_{1}=\left\{-4 \sum_{\gamma=1}^{3} \frac{B_{2 \gamma}}{(2 \gamma) !}\left[\int_{0}^{1} D_{x}^{2 \gamma-1} C_{1}(1, y) d y+\int_{0}^{1} D_{y}^{2 \gamma-1} C_{1}(x, 1) d x\right.\right. \\
& +\sum_{\mu=1}^{3} \frac{B_{2 \mu}}{(2 \mu) !} D_{x}^{2 \gamma-1} D_{y}^{2 \mu-1} C_{1}(1,1)+\int_{0}^{1} \hat{D}_{x}^{7} D_{y}^{2 \gamma-1} C_{1}(x, 1) d x \\
& \left.+\int_{0}^{1} \hat{D}_{y}^{7} D_{x}^{2 \gamma-1} C_{1}(1, y) d y\right]+4 \int_{1}^{\infty} \int_{0}^{\infty} \hat{D}_{x}^{7} C_{1}(x, y) d y d x \\
& +4 \int_{0}^{1} \int_{1}^{\infty} \hat{D}_{x}^{7} C_{1}(x, y) d y d x+4 \int_{1}^{\infty} \int_{0}^{\infty} \hat{D}_{y}^{7} C_{1}(x, y) d x d y \\
& +4 \int_{0}^{1} \int_{1}^{\infty} \hat{D}_{y}^{7} C_{1}(x, y) d x d y+4 \int_{1}^{\infty} \int_{0}^{\infty} \hat{D}_{x}^{7} \hat{D}_{y}^{7} C_{1}(x, y) d y d x \\
& \left.+4 \int_{0}^{1} \int_{1}^{\infty} \hat{D}_{x}^{7} \hat{D}_{y}^{7} C_{1}(x, y) d y d x+C_{1}(1,0)+C_{1}(1,1)-\int_{-1}^{1} \int_{-1}^{1} C_{1}(x, y) d x d y\right\} \\
& D_{3}=\left\{-4 \sum_{\gamma=1}^{3} \frac{B_{2 \gamma}}{(2 \gamma) !}\left[\int_{0}^{1} D_{x}^{2 \gamma-1} C_{3}(1, y) d y+\int_{0}^{1} D_{y}^{2 \gamma-1} C_{3}(x, 1) d x\right.\right. \\
& +\sum_{\mu=1}^{3} \frac{B_{2 \mu}}{(2 \mu) !} D_{x}^{2 \gamma-1} D_{y}^{2 \mu-1} C_{3}(1,1)+\int_{0}^{1} \hat{D}_{x}^{7} D_{y}^{2 \gamma-1} C_{3}(x, 1) d x \\
& \left.+\int_{0}^{1} \hat{D}_{y}^{7} D_{x}^{2 \gamma-1} C_{3}(1, y) d y\right]+4 \int_{1}^{\infty} \int_{0}^{\infty} \hat{D}_{x}^{7} C_{3}(x, y) d y d x \\
& +4 \int_{0}^{1} \int_{1}^{\infty} \int_{1}^{\infty} \hat{D}_{x}^{7} C_{3}(x, y) d y d x+4 \int_{1}^{\infty} \int_{0}^{\infty} \hat{D}_{y}^{7} C_{3}(x, y) d y d x+C_{3}(x, y) d x d y \\
& +4 \int_{0}^{1} \int_{1}^{\infty} \hat{D}_{y}^{7} C_{3}(x, y) d x d y+4 \int_{1}^{\infty} \int_{0}^{\infty} \hat{D}_{x}^{7} \hat{D}_{y}^{7} C_{3}(x, y) d y d x \\
& \left.+C_{3}(1,1)-\int_{-1}^{1} C_{-1}^{1} C_{3}(x, y) d x d y\right\}
\end{aligned}
$$

We have computed $D_{1}$ and $D_{3}$ to be approximately -.3103732 and $-1.33299 \times$ $10^{-3}$, respectively. These agree very well with the coefficients of the $h$ and $h^{3}$ terms 


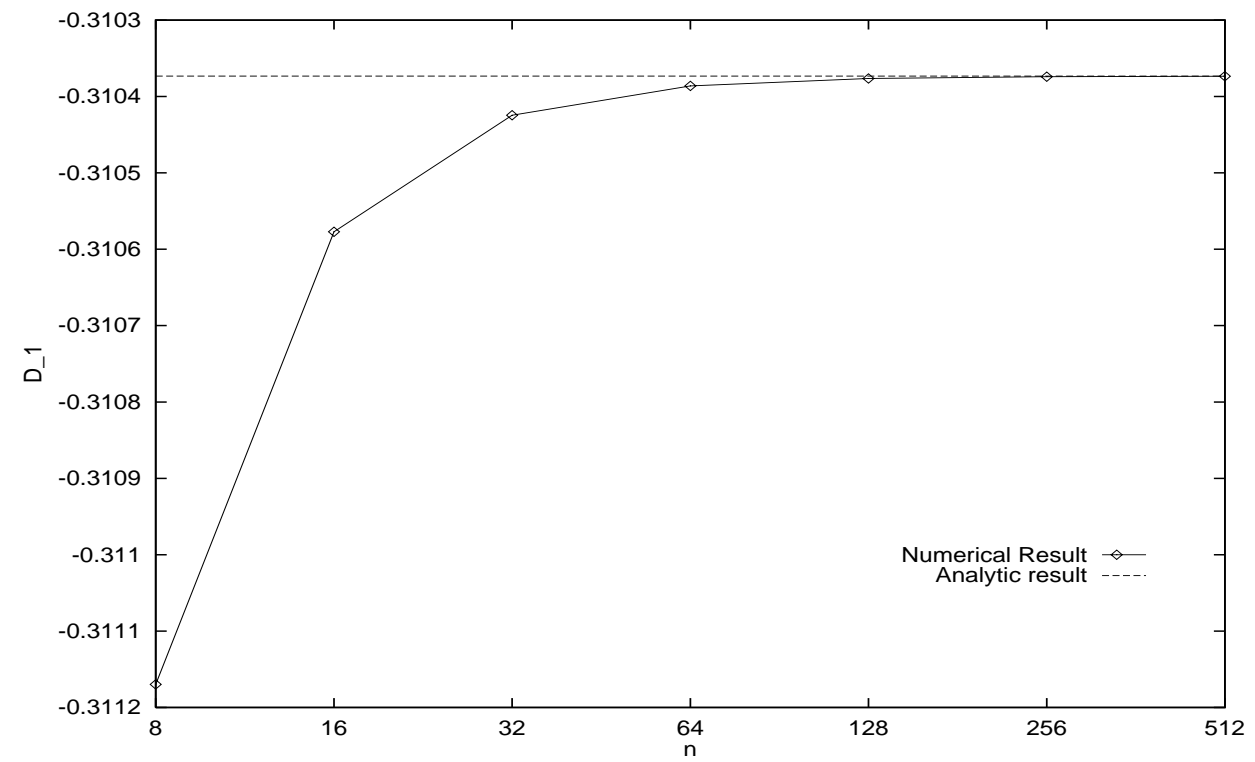

FIG. 4.1. The solid line represents the value of $D_{1}$ using (4.45). The dashed line represents the value of $D_{1}$ computed using the point-vortex approximation for various values of $n$.

computed from the point vortex calculation. Figure 4.1 demonstrates the comparison between the numerically computed value and the analytical value for $D_{1}$.

In the case of an interface that is not flat, the formula is similar but considerably more lengthy; we therefore omit the formula but sketch the approach. We can limit consideration to the case where $p(x, y)=x g(x, y)$ since the more general case is analogous. We expand $K(x, y) p(x, y)$ as outlined in section 4.1. Our analysis shows that the first term that contributes to the error expansion is $C_{1}$, which contains all of the terms which contribute to the first-order coefficient. We have

$$
\begin{aligned}
C_{1}= & \frac{x\left[g_{x}(0,0) x+g_{y}(0,0) y\right]}{\left(A x^{2}+B y^{2}+C x y\right)^{3 / 2}} \\
& -\frac{3}{2} x g(0,0) \frac{\left[\eta_{x}(0,0) x+\eta_{y}(0,0) y\right]\left[\eta_{x x}(0,0) x^{2}+\eta_{y y}(0,0) y^{2}+2 \eta_{x y}(0,0) x y\right]}{\left(A x^{2}+B y^{2}+C x y\right)^{5 / 2}},
\end{aligned}
$$

where

$$
\begin{aligned}
& A=1+\eta_{x}^{2}(0,0) \\
& B=1+\eta_{y}^{2}(0,0) \\
& C=2 \eta_{x}(0,0) \eta_{y}(0,0) .
\end{aligned}
$$

For a specific example, we take

$$
\begin{aligned}
\gamma_{x} & =-2(\sin (x)+\cos (x)), \\
\eta(x, y) & =\sin (x+y)+\cos (x+y), \\
\gamma_{y} & =0 .
\end{aligned}
$$

From $C_{1}$, we compute $D_{1}$ to be approximately -.21733 . This gives good agreement with the value we computed using the point vortex method. 


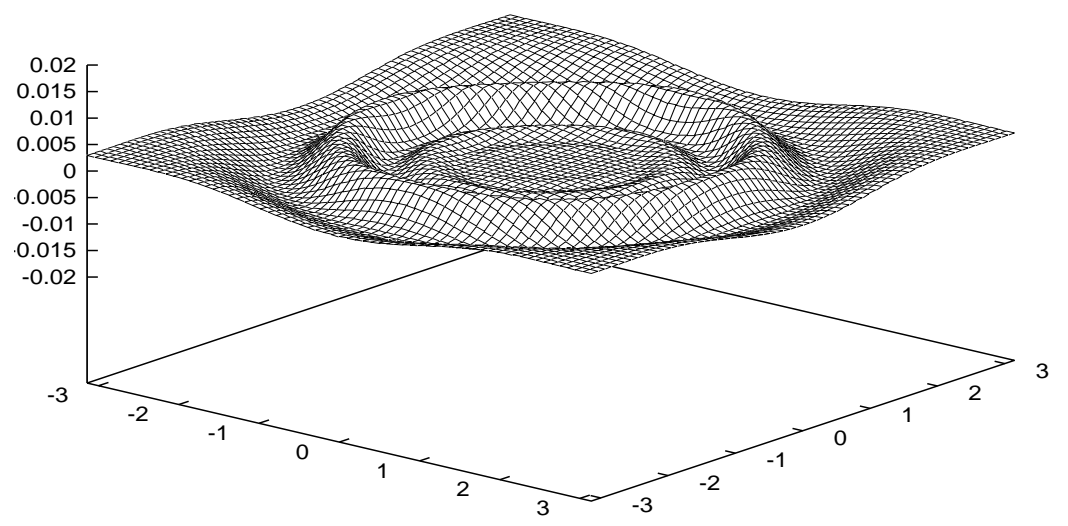

FIG. 5.1. Wave computed using a Gaussian for the initial wave profile.

5. Conclusion. In the preceding analysis, we have been considering only one component of velocity. The extension of this method to the other components of velocity is straightforward. We note that this method also predicts that the pointvortex approximation to the potential $\phi$ should be a series in odd powers of $h$, which we have verified numerically. The direct application of the two-dimensional point vortex method is impractical from a numerical standpoint because the operation count in calculating the velocity field is $O\left(n^{4}\right)$, where $n$ is the number of points in one dimension. One method which we have used for decreasing the order of operations is the fast multipole method (see Greengard [13]). In this case the operation count reduces to $O\left(n^{2}\right)$. We will discuss the fast multipole implementation and results in a future paper.

As a test of the method, we have computed the motion of a two-dimensional Stokes wave but using a three-dimensional vortex sheet. We used a third-order point vortex method to compute the velocities and used fourth-order Adams-Bashforth to perform the time evolution. The Stokes wave in our example had an amplitude of approximately .2356, which is about $53 \%$ of the maximum amplitude for Stokes waves. We chose $n=32$ and propagated the wave for approximately one quarter of a wavelength. The computed solution agrees well with the analytical solution. Specifically, the numerical solution preserves the wave profile.

We have also computed the motion of a three-dimensional wave where the initial profile is a Gaussian. The form of the Gaussian is

$$
\eta(x, y, 0)=\frac{e^{-10\left(x^{2}+y^{2}\right)}}{20}
$$

on the region $[-2 \pi, 2 \pi] \times[-2 \pi, 2 \pi]$ and is extended periodically in each dimension. We chose $n=64$ (corresponding to a $64 \times 64$ mesh) and $\Delta t=.01$ and propagated the wave for several hundred time steps. We show the result for $t=9.5$ in Figure 5.1.

Appendix A. Two-dimensional Euler-Maclaurin summation formula. Before deriving the formula, we make the following definitions:

$$
\begin{aligned}
w_{i} & =\left\{\begin{aligned}
\frac{1}{2} & \text { if } i=0 \text { or } i=n, \\
1 & \text { otherwise, }
\end{aligned}\right. \\
D_{x}^{j} & \equiv \frac{\partial^{j}}{\partial x^{j}}, \quad \tilde{D}_{x}^{j} \equiv P_{2 m+1}(x / h) D_{x}^{j} .
\end{aligned}
$$


The function $P_{m}(x)$ is given by

$$
P_{m}(x)=\frac{\bar{B}_{m}(x)}{m !},
$$

where $\bar{B}_{m}(x)$ is the Bernoulli polynomial of order $m$ on $[0,1]$ extended periodically. $B_{i}$ is the $i$ th Bernoulli number. The two-dimensional version of the Euler-Maclaurin summation formula is derived as below. First apply the one-dimensional formula in the $y$ direction:

$$
\begin{aligned}
\int_{a}^{b} \int_{c}^{d} f(x, y) d x d y=\int_{a}^{b} & {\left[h \sum_{j=0}^{n} w_{j} f\left(x, y_{j}\right)-\sum_{\mu=1}^{m} \frac{h^{2 \mu} B_{2 \mu}}{(2 \mu) !}\left[D_{y}^{2 \mu-1} f(x, d)\right.\right.} \\
& \left.\left.-D_{y}^{2 \mu-1} f(x, c)\right]-h^{2 m+1} \int_{c}^{d} \tilde{D}_{y}^{2 m+1} f(x, y) d y\right] d x .
\end{aligned}
$$

Next, we apply the trapezoidal rule in the $x$ direction for the first sum term, and explicitly integrate the other terms:

$$
\begin{aligned}
& \int_{a}^{b} \int_{c}^{d} f(x, y) d x d y=h^{2} \sum_{i=0}^{n} \sum_{j=0}^{n} w_{i} w_{j} f\left(x_{i}, y_{j}\right) \\
& -\sum_{j=0}^{n} \sum_{\gamma=1}^{m} \frac{h^{2 \gamma+1} B_{2 \gamma}}{(2 \gamma) !}\left[D_{x}^{2 \gamma-1} w_{j} f\left(b, y_{j}\right)-D_{x}^{2 \gamma-1} w_{j} f\left(a, y_{j}\right)\right] \\
& -h \sum_{j=0}^{n} h^{2 m+1} \int_{a}^{b} \tilde{D}_{x}^{2 m+1} w_{j} f\left(x, y_{j}\right) d x \\
& -\sum_{\mu=1}^{m} \frac{h^{2 \mu} B_{2 \mu}}{(2 \mu) !}\left[\int_{a}^{b}\left[D_{y}^{2 \mu-1} f(x, d)-D_{y}^{2 \mu-1} f(x, c)\right] d x\right] \\
& -h^{2 m+1} \int_{c}^{d} \int_{a}^{b} \tilde{D}_{y}^{2 m+1} f(x, y) d x d y .
\end{aligned}
$$

In the second and third terms of this equation, the sum over $j$ represents a trapezoidal rule integration, so we can apply the 1-d formula in each of these terms to give onedimensional

$$
\begin{aligned}
& \int_{a}^{b} \int_{c}^{d} f(x, y) d x d y=h^{2} \sum_{i=0}^{n} \sum_{j=0}^{n} w_{i} w_{j} f\left(x_{i}, y_{j}\right) \\
& -\sum_{\gamma=1}^{m} \frac{h^{2 \gamma} B_{2 \gamma}}{(2 \gamma) !}\left[\int_{c}^{d}\left[D_{x}^{2 \gamma-1} f(b, y)-D_{x}^{2 \gamma-1} f(a, y)\right] d y\right. \\
& +\sum_{\mu=1}^{m} \frac{h^{2 \mu} B_{2 \mu}}{(2 \mu) !}\left[D_{x}^{2 \gamma-1} D_{y}^{2 \mu-1} f(b, d)-D_{x}^{2 \gamma-1} D_{y}^{2 \mu-1} f(b, c)\right. \\
& \left.-D_{x}^{2 \gamma-1} D_{y}^{2 \mu-1} f(a, d)+D_{x}^{2 \gamma-1} D_{y}^{2 \mu-1} f(a, c)\right] \\
& \left.+h^{2 m+1} \int_{c}^{d} \tilde{D}_{y}^{2 m+1}\left[D_{x}^{2 \gamma-1} f(b, y)-D_{x}^{2 \gamma-1} f(a, y)\right] d y\right] \\
& -h^{2 m+1} \int_{a}^{b} \int_{c}^{d} \tilde{D}_{x}^{2 m+1} f(x, y) d y d x
\end{aligned}
$$




$$
\begin{aligned}
& -h^{2 m+1} \int_{a}^{b} \sum_{\gamma=1}^{m} \frac{h^{2 \gamma} B_{2 \gamma}}{(2 \gamma) !} \tilde{D}_{x}^{2 m+1}\left[D_{y}^{2 \gamma-1} f(x, d)-D_{y}^{2 \gamma-1} f(x, c)\right] d x \\
& -h^{4 m+2} \int_{a}^{b} \int_{c}^{d} \tilde{D}_{x}^{2 m+1} \tilde{D}_{y}^{2 m+1} f(x, y) d x d y \\
& -\sum_{\mu=1}^{m} \frac{h^{2 \mu} B_{2 \mu}}{(2 \mu) !} \int_{a}^{b}\left[D_{y}^{2 \mu-1} f(x, d)-D_{y}^{2 \mu-1} f(x, c)\right] d x \\
& -h^{2 m+1} \int_{c}^{d} \int_{a}^{b} \tilde{D}_{y}^{2 m+1} f(x, y) d x d y .
\end{aligned}
$$

Regrouping the terms gives

$$
\begin{aligned}
& h^{2} \sum_{i=0}^{n} \sum_{j=0}^{n} w_{i} w_{j} f\left(x_{i}, y_{j}\right)-\int_{a}^{b} \int_{c}^{d} f(x, y) d x d y \\
& =\sum_{\gamma=1}^{m} \frac{h^{2 \gamma} B_{2 \gamma}}{(2 \gamma) !}\left[\int_{c}^{d}\left[D_{x}^{2 \gamma-1} f(b, y)-D_{x}^{2 \gamma-1} f(a, y)\right] d y+\int_{a}^{b}\left[D_{y}^{2 \gamma-1} f(x, d)\right.\right. \\
& \left.-D_{y}^{2 \gamma-1} f(x, c)\right] d x+\sum_{\mu=1}^{m} \frac{h^{2 \mu} B_{2 \mu}}{(2 \mu) !}\left[D_{x}^{2 \gamma-1} D_{y}^{2 \mu-1} f(b, d)\right. \\
& \left.-D_{x}^{2 \gamma-1} D_{y}^{2 \mu-1} f(b, c)-D_{x}^{2 \gamma-1} D_{y}^{2 \mu-1} f(a, d)+D_{x}^{2 \gamma-1} D_{y}^{2 \mu-1} f(a, c)\right] \\
& +h^{2 m+1} \int_{a}^{b} \tilde{D}_{x}^{2 m-1}\left[D_{y}^{2 \gamma+1} f(x, d)-D_{y}^{2 \gamma-1} f(x, c)\right] d x \\
& \left.+h^{2 m+1} \int_{c}^{d} \tilde{D}_{y}^{2 m+1}\left[D_{x}^{2 \gamma-1} f(b, y)-D_{x}^{2 \gamma-1} f(a, y)\right] d y\right] \\
& +h^{2 m+1} \int_{a}^{b} \int_{c}^{d} \tilde{D}_{x}^{2 m+1} f(x, y) d y d x+h^{2 m+1} \int_{c}^{d} \int_{a}^{b} \tilde{D}_{y}^{2 m+1} f(x, y) d x d y \\
& +h^{4 m+2} \int_{a}^{b} \int_{c}^{d} \tilde{D}_{x}^{2 m+1} \tilde{D}_{y}^{2 m+1} f(x, y) d y d x .
\end{aligned}
$$

A.1. Calculation of the error. In order to apply (A.6), we rewrite $I$ as a sum of singular and nonsingular integrals, $I=I_{s}+I_{r}$. We can then also write the error as $E=E_{s}+E_{r}$, where $E_{s}$ is the error associated with the approximation to $I_{s}$ and $E_{r}$ is the error associated with $I_{r}$. We can write $I$ as

$$
I=I_{s}+I_{r}=I_{s}+\sum_{i=1}^{4} I_{r_{i}},
$$

where

$$
\begin{aligned}
I_{s} & =\int_{-\pi}^{\pi} \int_{-\pi}^{\pi} f(x, y) d x d y, \\
I_{r_{1}} & =\int_{-\infty}^{\pi} \int_{-\pi}^{\pi} f(x, y) d x d y, \\
I_{r_{2}} & =\int_{\pi}^{\infty} \int_{-\pi}^{\pi} f(x, y) d x d y,
\end{aligned}
$$




$$
\begin{aligned}
I_{r_{3}} & =\int_{-\infty}^{\infty} \int_{-\infty}^{-\pi} f(x, y) d x d y \\
I_{r_{4}} & =\int_{-\infty}^{\infty} \int_{\pi}^{\infty} f(x, y) d x d y
\end{aligned}
$$

If we define $\Omega$ by

$$
\Omega=\mathbb{R}^{2} /\{[-\pi, \pi] \times[-\pi, \pi]\}
$$

then we have

$$
I_{r}=\iint_{\Omega} f(x, y) d x d y
$$

We denote the trapezoidal rule approximation to $I_{r_{i}}$ by $I_{A_{i}}$ and the trapezoidal rule approximation to $I_{s}$ by $I_{A_{s}}$. We define the respective errors as $E_{r_{i}}=I_{A_{i}}-I_{r_{i}}$ and $E_{s}=I_{A_{s}}-I_{s}$. Finally, we define $E_{r}=\sum_{i=1}^{4} E_{r_{i}}$.

A.2. Calculation of $\boldsymbol{E}_{\boldsymbol{r}}$. We can use the two-dimensional Euler-Maclaurin formula to derive the following result for $E_{r_{1}}$ :

$$
\begin{aligned}
E_{r_{1}}= & I_{A_{1}}-I_{r_{1}} \\
& =\sum_{\gamma=1}^{m} \frac{h^{2 \gamma} B_{2 \gamma}}{(2 \gamma) !}\left[\int_{-\pi}^{\pi} D_{x}^{2 \gamma-1} f(-\pi, y) d y\right. \\
& +\int_{-\infty}^{-\pi}\left[D_{y}^{2 \gamma-1} f(x, \pi)-D_{y}^{2 \gamma-1} f(x,-\pi)\right] d x \\
& +\sum_{\mu=1}^{m} \frac{h^{2 \mu} B_{2 \mu}}{(2 \mu) !}\left[D_{x}^{2 \gamma-1} D_{y}^{2 \mu-1} f(-\pi, \pi)-D_{x}^{2 \gamma-1} D_{y}^{2 \mu-1} f(-\pi,-\pi)\right] \\
& +h^{2 m+1} \int_{-\infty}^{-\pi} \tilde{D}_{x}^{2 m+1}\left[D_{y}^{2 \gamma-1} f(x, \pi)-D_{y}^{2 \gamma-1} f(x,-\pi)\right] d x \\
& \left.+h^{2 m+1} \int_{-\pi}^{\pi} \tilde{D}_{y}^{2 m+1} D_{x}^{2 \gamma-1} f(-\pi, y) d y\right] \\
& +h^{2 m+1} \int_{-\infty}^{-\pi} \int_{-\pi}^{\pi} \tilde{D}_{x}^{2 m+1} f(x, y) d y d x+h^{2 m+1} \int_{-\pi}^{\pi} \int_{-\infty}^{-\pi} \tilde{D}_{y}^{2 m+1} f(x, y) d x d y \\
& +h^{4 m+2} \int_{-\infty}^{-\pi} \int_{-\pi}^{\pi} \tilde{D}_{x}^{2 m+1} \tilde{D}_{y}^{2 m+1} f_{x y}^{2 m+1,2 m+1}(x, y) d y d x .
\end{aligned}
$$

We can derive a similar result for each of the other three regular integrals. We then add all of the terms together to get

$$
\begin{aligned}
& E_{r}=\sum_{i=1}^{4}\left(I_{A_{i}}-I_{r_{i}}\right)=\sum_{\gamma=1}^{m} \frac{h^{2 \gamma} B_{2 \gamma}}{(2 \gamma) !}\left[\int_{-\pi}^{\pi}\left[D_{x}^{2 \gamma-1} f(-\pi, y)-D_{x}^{2 \gamma-1} f(\pi, y)\right] d y\right. \\
& +\int_{-\pi}^{\pi}\left[D_{y}^{2 \gamma-1} f(x,-\pi)-D_{y}^{2 \gamma-1} f(x, \pi)\right] d x+\sum_{\mu=1}^{m} \frac{h^{2 \mu} B_{2 \mu}}{(2 \mu) !}\left[D_{x}^{2 \gamma-1} D_{y}^{2 \mu-1} f(-\pi, \pi)\right.
\end{aligned}
$$




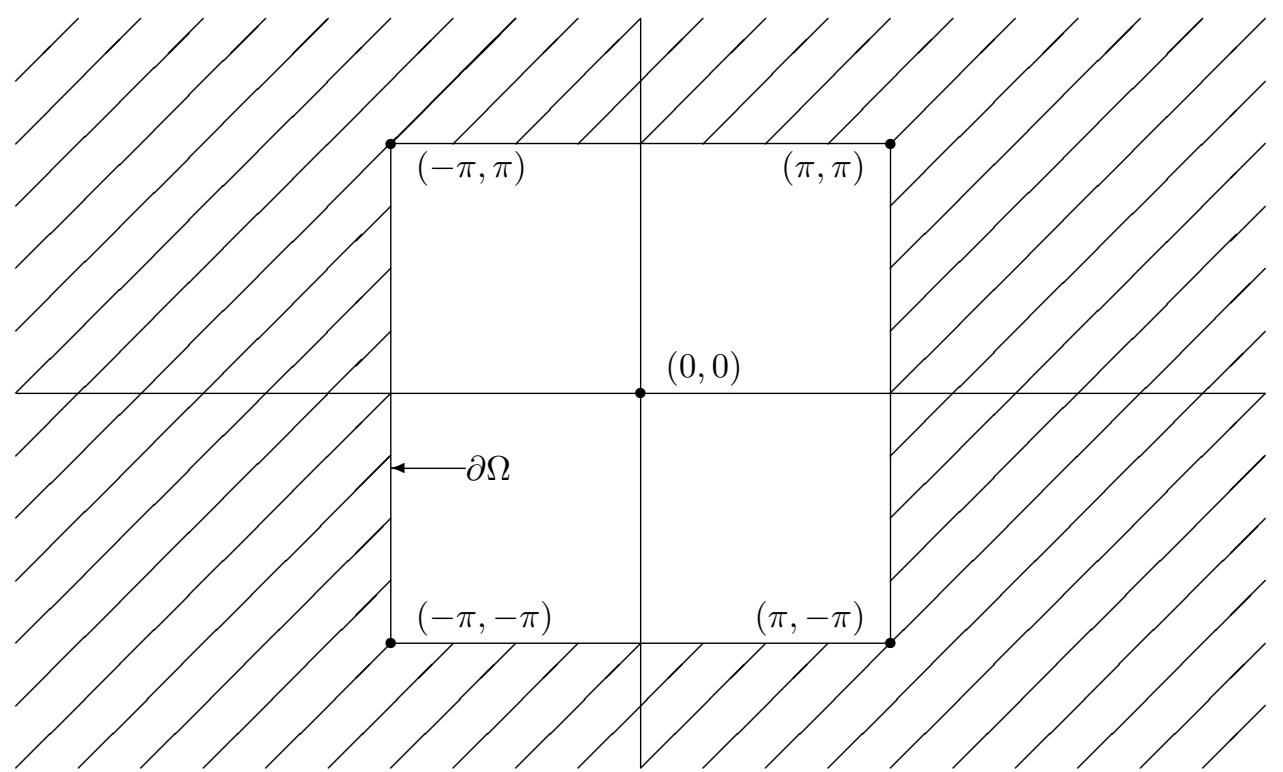

FIG. A.1. The shaded region is $\Omega$ and extends infinitely in both dimensions. The boundary is $\partial \Omega$.

$$
\begin{aligned}
& \left.-D_{x}^{2 \gamma-1} D_{y}^{2 \mu-1} f(-\pi,-\pi)-D_{x}^{2 \gamma-1} D_{y}^{2 \mu-1} f(\pi, \pi)+D_{x}^{2 \gamma-1} D_{y}^{2 \mu-1} f(\pi,-\pi)\right] \\
& +h^{2 m+1} \int_{-\pi}^{\pi} \tilde{D}_{x}^{2 m+1}\left[D_{y}^{2 \gamma-1} f(x,-\pi)-D_{y}^{2 \gamma-1} f(x, \pi)\right] d x \\
& \left.+h^{2 m+1} \int_{-\pi}^{\pi} \tilde{D}_{y}^{2 m+1}\left[D_{x}^{2 \gamma-1} f(-\pi, y)-D_{x}^{2 \gamma-1} f(\pi, y)\right] d y\right] \\
& +h^{2 m+1} \iint_{\Omega} \tilde{D}_{x}^{2 m+1} f(x, y) d y d x \\
& +h^{2 m+1} \iint_{\Omega} \tilde{D}_{y}^{2 m+1} f(x, y) d y d x \\
& +h^{4 m+2} \iint_{\Omega} \tilde{D}_{x}^{2 m+1} \tilde{D}_{y}^{2 m+1} f(x, y) d y d x .
\end{aligned}
$$

We define $\partial \Omega$ to be the boundary of $\Omega$ as shown in Figure A.1. The last three terms in the previous expression are integrals over $\Omega$. We denote these terms by $E_{\Omega}$. The rest of the terms in the expression are evaluated in terms of $\partial \Omega$. We denote these terms by $-E_{\partial \Omega}$ (we use the negative sign for convenience).

Thus we have

$$
E_{r}=-E_{\partial \Omega}+E_{\Omega}
$$

where

$$
\begin{aligned}
& E_{\partial \Omega}=-\sum_{\gamma=1}^{m} \frac{h^{2 \gamma} B_{2 \gamma}}{(2 \gamma) !}\left[\int_{-\pi}^{\pi}\left[D_{x}^{2 \gamma-1} f(-\pi, y)-D_{x}^{2 \gamma-1} f(\pi, y)\right] d y\right. \\
& +\int_{-\pi}^{\pi}\left[D_{y}^{2 \gamma-1} f(x,-\pi)-D_{y}^{2 \gamma-1} f(x, \pi)\right] d x+\sum_{\mu=1}^{m} \frac{h^{2 \mu} B_{2 \mu}}{(2 \mu) !}\left[D_{x}^{2 \gamma-1} D_{y}^{2 \mu-1} f(-\pi, \pi)\right.
\end{aligned}
$$




$$
\begin{aligned}
& \left.-D_{x}^{2 \gamma-1} D_{y}^{2 \mu-1} f(-\pi,-\pi)-D_{x}^{2 \gamma-1} D_{y}^{2 \mu-1} f(\pi, \pi)+D_{x}^{2 \gamma-1} D_{y}^{2 \mu-1} f(\pi,-\pi)\right] \\
& +h^{2 m+1} \int_{-\pi}^{\pi} \tilde{D}_{x}^{2 m+1}\left[D_{y}^{2 \gamma-1} f(x,-\pi)-D_{y}^{2 \gamma-1} f(x, \pi)\right] d x \\
& \left.+h^{2 m+1} \int_{-\pi}^{\pi} \tilde{D}_{y}^{2 m+1}\left[D_{x}^{2 \gamma-1} f(-\pi, y)-D_{x}^{2 \gamma-1} f(\pi, y)\right] d y\right]
\end{aligned}
$$

and

$$
\begin{aligned}
E_{\Omega}= & h^{2 m+1} \iint_{\Omega} \tilde{D}_{x}^{2 m+1} f(x, y) d y d x \\
& +h^{2 m+1} \iint_{\Omega} \tilde{D}_{y}^{2 m+1} f(x, y) d y d x \\
& +h^{4 m+2} \iint_{\Omega} \tilde{D}_{x}^{2 m+1} \tilde{D}_{y}^{2 m+1} f(x, y) d y d x .
\end{aligned}
$$

We note that $E_{\partial \Omega}$ is a finite series in even powers of $h$. We comment later on $E_{\Omega}$.

A.3. Calculation of $\boldsymbol{E}_{\boldsymbol{s}}$. We now consider the error that comes from applying the trapezoidal rule to the singular integral $I_{s}$. Because of the singularity, we must use the point vortex-method. We can write

$$
E_{s}=I_{A_{s}}-I_{s}=\sum_{i=-n / 2}^{n / 2} \sum_{\substack{j=-n / 2 \\(i, j) \neq 0}}^{n / 2} w_{i} w_{j} f(i h, j h)-\int_{-\pi}^{\pi} \int_{-\pi}^{\pi} f(x, y) d x d y
$$

We want to express $E_{s}$ in a form which allows the direct use of the Euler-Maclaurin formula. To do this, we break $I_{s}$ and $I_{A_{s}}$ into several integrals and sums and arrange them as follows:

$$
E_{s}=E_{1}+E_{2}+E_{3}+E_{4}+E_{5},
$$

where

$$
\begin{aligned}
& E_{1}=h^{2} \sum_{i=-n / 2}^{-1} \sum_{j=-n / 2}^{n / 2} \tilde{w}_{i} \tilde{w}_{j} f(i h, j h)-\int_{-\pi}^{-h} \int_{-\pi}^{\pi} f(x, y) d y d x, \\
& E_{2}=h^{2} \sum_{i=1}^{n / 2} \sum_{j=-n / 2}^{n / 2} \tilde{w}_{i} \tilde{w}_{j} f(i h, j h)-\int_{h}^{\pi} \int_{-\pi}^{\pi} f(x, y) d y d x, \\
& E_{3}=h^{2} \sum_{i=-1}^{1} \sum_{j=1}^{n / 2} \tilde{w}_{i} \tilde{w}_{j} f(i h, j h)-\int_{-h}^{h} \int_{h}^{\pi} f(x, y) d y d x, \\
& E_{4}=h^{2} \sum_{i=-1}^{1} \sum_{j=-n / 2}^{-1} \tilde{w}_{i} \tilde{w}_{j} f(i h, j h)-\int_{-h}^{h} \int_{-\pi}^{-h} f(x, y) d y d x, \\
& E_{5}=\sum_{i=-1}^{1} \sum_{\substack{j=-1 \\
(i, j) \neq(0,0)}}^{1} \tilde{w}_{i} \tilde{w}_{j} f(i h, j h)-\int_{-h}^{h} \int_{-h}^{h} f(x, y) d y d x .
\end{aligned}
$$


The factor $\tilde{w}_{i}$ is defined by

$$
\tilde{w}_{i}= \begin{cases}\frac{1}{2} & \text { if } i=i_{\min } \text { or } i=i_{\max }, \\ 1 & \text { otherwise, }\end{cases}
$$

where $i_{\max }$ and $i_{\min }$ are the respective upper and lower limits of the summation index $i$. For example, $i_{\max }=-1$ and $i_{\min }=-n / 2$ in the equation defining $E_{1}$. Using the two-dimensional Euler-Maclaurin formula, we can evaluate $E_{1}$ through $E_{4}$. After some manipulation, we arrive at the following result:

$$
\begin{aligned}
& E_{1}+E_{2}+E_{3}+E_{4}=-\sum_{\gamma=1}^{m} \frac{h^{2 \gamma} B_{2 \gamma}}{(2 \gamma) !}\left[\int_{-\pi}^{\pi}\left[D_{x}^{2 \gamma-1} f(-\pi, y)-D_{x}^{2 \gamma-1} f(\pi, y)\right] d y\right. \\
& +\int_{-\pi}^{\pi}\left[D_{y}^{2 \gamma-1} f(x,-\pi)-D_{y}^{2 \gamma-1} f(x, \pi)\right] d x+\sum_{\mu=1}^{m} \frac{h^{2 \mu} B_{2 \mu}}{(2 \mu) !}\left[D_{x}^{2 \gamma-1} D_{y}^{2 \mu-1} f(-\pi, \pi)\right. \\
& \left.-D_{x}^{2 \gamma-1} D_{y}^{2 \mu-1} f(-\pi,-\pi)-D_{x}^{2 \gamma-1} D_{y}^{2 \mu-1} f(\pi, \pi)+D_{x}^{2 \gamma-1} D_{y}^{2 \mu-1} f(\pi,-\pi)\right] \\
& +h^{2 m+1} \int_{-\pi}^{\pi} \tilde{D}_{x}^{2 m+1}(x / h)\left[D_{y}^{2 \gamma-1} f(x,-\pi)-D_{y}^{2 \gamma-1} f(x, \pi)\right] d x \\
& \left.+h^{2 m+1} \int_{-\pi}^{\pi} \tilde{D}_{y}^{2 m+1}\left[D_{x}^{2 \gamma-1} f(-\pi, y)-D_{x}^{2 \gamma-1} f(\pi, y)\right] d y\right] \\
& -\sum_{\gamma=1}^{m} \frac{h^{2 \gamma} B_{2 \gamma}}{(2 \gamma) !}\left[\int_{-h}^{h}\left[D_{x}^{2 \gamma-1} f(h, y)-D_{x}^{2 \gamma-1} f(-h, y)\right] d y\right. \\
& +\int_{-h}^{h}\left[D_{y}^{2 \gamma-1} f(x, h)-D_{y}^{2 \gamma-1} f(x,-h)\right] d x+\sum_{\mu=1}^{m} \frac{h^{2 \mu} B_{2 \mu}}{(2 \mu !)}\left[D_{x}^{2 \gamma-1} D_{y}^{2 \mu-1} f(h, h)\right. \\
& \left.-D_{x}^{2 \gamma-1} D_{y}^{2 \mu-1} f(-h, h)-D_{x}^{2 \gamma-1} D_{y}^{2 \mu-1} f(h,-h)+D_{x}^{2 \gamma-1} D_{y}^{2 \mu-1} f(-h,-h)\right] \\
& +h^{2 m+1} \int_{-h}^{h} \tilde{D}_{x}^{2 m+1}\left[D_{y}^{2 \gamma-1} f(x, h)-D_{y}^{2 \gamma-1} f(x,-h)\right] d x \\
& \left.+h^{2 m+1} \int_{-h}^{h} \tilde{D}_{y}^{2 m+1}\left[D_{x}^{2 \gamma-1} f(h, y)-D_{x}^{2 \gamma-1} f(-h, y)\right] d y\right] \\
& +h^{2 m+1} \int_{\Lambda} \tilde{D}_{x}^{2 m+1} f(x, y) d x d y+h^{2 m+1} \iint_{\Lambda} \tilde{D}_{y}^{2 m+1} f(x, y) d x d y \\
& +h^{4 m+2} \int_{\Lambda} \tilde{D}_{x}^{2 m+1} \tilde{D}_{y}^{2 m+1} f(x, y) d x d y, \\
& \\
& \quad{ }^{2 m} f(x)
\end{aligned}
$$

where

$$
\Lambda=\{[-\pi, \pi] \times[-\pi, \pi]\} /\{[-h, h] \times[-h, h]\} .
$$

We define the inner boundary of $\Lambda$ to be $\partial \Lambda$ and note that the outer boundary of $\Lambda$ is $\partial \Omega$, the inner boundary of $\Omega$, as shown in Figure A.2. We define $E_{\partial \Lambda}$ and $E_{\Lambda}$ by

$$
E_{\partial \Lambda}=\sum_{\gamma=1}^{m} \frac{h^{2 \gamma} B_{2 \gamma}}{(2 \gamma) !}\left[\int_{-h}^{h}\left[D_{x}^{2 \gamma-1} f(h, y)-D_{x}^{2 \gamma-1} f(-h, y)\right] d y\right.
$$




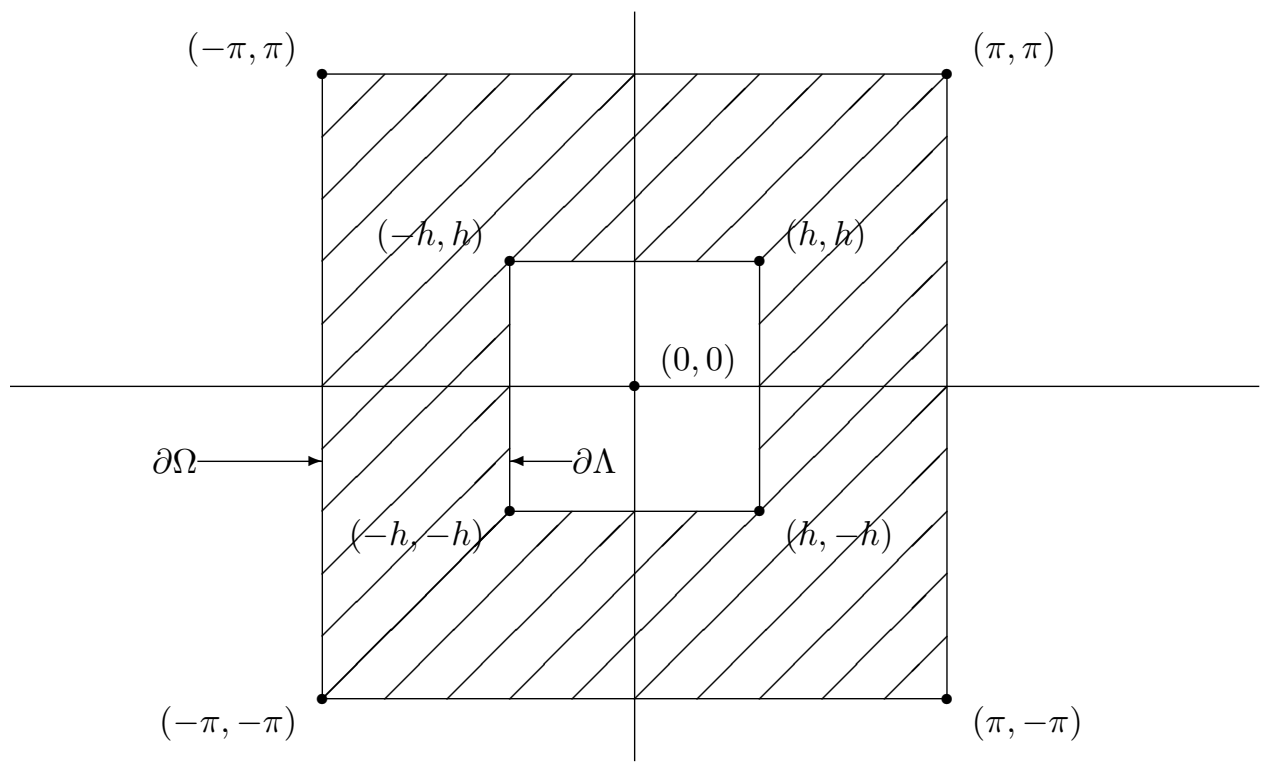

FIG. A.2. The shaded region is $\Lambda$. The inner boundary is $\partial \Lambda$. The outer boundary is $\partial \Omega$.

$$
\begin{aligned}
& +\int_{-h}^{h}\left[D_{y}^{2 \gamma-1} f(x, h)-D_{y}^{2 \gamma-1} f(x,-h)\right] d x+\sum_{\mu=1}^{m} \frac{h^{2 \mu} B_{2 \mu}}{(2 \mu !)}\left[D_{x}^{2 \gamma-1} D_{y}^{2 \mu-1} f(h, h)\right. \\
& \left.-D_{x}^{2 \gamma-1} D_{y}^{2 \mu-1} f(-h, h)-D_{x}^{2 \gamma-1} D_{y}^{2 \mu-1} f(h,-h)+D_{x}^{2 \gamma-1} D_{y}^{2 \mu-1} f(-h,-h)\right] \\
& +h^{2 m+1} \int_{-h}^{h} \tilde{D}_{x}^{2 m+1}\left[D_{y}^{2 \gamma-1} f(x, h)-D_{y}^{2 \gamma-1} f(x,-h)\right] d x \\
& \left.+h^{2 m+1} \int_{-h}^{h} \tilde{D}_{y}^{2 m+1}\left[D_{x}^{2 \gamma-1} f(h, y)-D_{y}^{2 \gamma-1} f(-h, y)\right] d y\right]
\end{aligned}
$$

and

$$
\begin{aligned}
E_{\Lambda}= & h^{2 m+1} \iint_{\Lambda} \tilde{D}_{x}^{2 m+1} f(x, y) d x d y+h^{2 m+1} \iint_{\Lambda} \tilde{D}_{y}^{2 m+1} f(x, y) d x d y \\
& +h^{4 m+2} \iint_{\Lambda} \tilde{D}_{x}^{2 m+1} \tilde{D}_{y}^{2 m+1} f(x, y) d x d y
\end{aligned}
$$

Using the definitions of $E_{\partial \Lambda}, E_{\Lambda}$, and $E_{\partial \Omega}$ (recall (A.15)), we see that we can write

$$
E_{1}+E_{2}+E_{3}+E_{4}=E_{\partial \Omega}-E_{\partial \Lambda}+E_{\Lambda}
$$

We can now write a simpler expression for $E_{s}$ :

$$
\begin{aligned}
E_{s} & =E_{1}+E_{2}+E_{3}+E_{4}+E_{5} \\
& =E_{\partial \Omega}-E_{\partial \Lambda}+E_{\Lambda}+E_{5} .
\end{aligned}
$$


A.4. Formula for $\boldsymbol{E}$. Putting together our definitions of $E_{r}$ and $E_{s}$, we can now write an expression for $E$ :

$$
\begin{aligned}
E & =E_{s}+E_{r} \\
& =-E_{\partial \Omega}+E_{\Omega}+E_{\partial \Omega}+E_{\Lambda}-E_{\partial \Lambda}+E_{5} \\
& =E_{\Omega}+E_{\Lambda}-E_{\partial \Lambda}+E_{5} \\
& =E_{\Omega}+E_{L},
\end{aligned}
$$

where

$$
E_{L}=E_{\Lambda}-E_{\partial \Lambda}+E_{5} .
$$

We show that $E_{L}$ is a series in odd powers of $h$. It is significant that $E_{\partial \Omega}$ does not appear in $E$, as this means that there are no even powers of $h$ in $E$. We show later that

$$
E_{\Omega}=o\left(h^{2 m}\right) .
$$

To simplify the computation of $E_{L}$, we use the notation $E_{L}(f)$ to mean that we plug $f$ into the expression for $E_{L}$ and we observe that $E_{L}$ is linear. That is, if $a$ and $b$ are constants and $f$ and $g$ are functions of $x$ and $y$, then $E_{L}(a f+b g)=$ $a E_{L}(f)+b E_{L}(g)$. Using this notation, the definition of $E_{L}$ (A.31), and the definition of $\Lambda$ (A.26), we have

$$
\begin{aligned}
& E_{L}(f)=h^{2 m+1} \iint_{\Lambda} \tilde{D}_{x}^{2 m+1} f(x, y) d x d y+h^{2 m+1} \iint_{\Lambda} D_{y}^{2 m+1} f(x, y) d x d y \\
& +h^{4 m+2} \iint_{\Lambda} \tilde{D}_{x}^{2 m+1} \tilde{D}_{y}^{2 m+1} f(x, y) d x d y \\
& -\sum_{\gamma=1}^{m} \frac{h^{2 \gamma} B_{2 \gamma}}{(2 \gamma) !}\left[\int_{-h}^{h}\left[D_{x}^{2 \gamma-1} f(h, y)-D_{x}^{2 \gamma-1} f(-h, y)\right] d y\right. \\
& +\int_{-h}^{h}\left[D_{y}^{2 \gamma-1} f(x, h)-D_{y}^{2 \gamma-1} f(x,-h)\right] d x+\sum_{\mu=1}^{m} \frac{h^{2 \mu} B_{2 \mu}}{(2 \mu !)}\left[D_{x}^{2 \gamma-1} D_{y}^{2 \mu-1} f(h, h)\right. \\
& \left.-D_{x}^{2 \gamma-1} D_{y}^{2 \mu-1} f(-h, h)-D_{x}^{2 \gamma-1} D_{y}^{2 \mu-1} f(h,-h)+D_{x}^{2 \gamma-1} D_{y}^{2 \mu-1} f(-h,-h)\right] \\
& -h^{2 m+1} \int_{-h}^{h} \tilde{D}_{x}^{2 m+1}\left[D_{y}^{2 \gamma-1} f(x, h)-D_{y}^{2 \gamma-1} f(x,-h)\right] d x \\
& \left.-h^{2 m+1} \int_{-h}^{h} \tilde{D}_{y}^{2 m+1}\left[D_{x}^{2 \gamma-1} f(h, y)-D_{y}^{2 \gamma-1} f(-h, y)\right] d y\right] \\
& +\sum_{i=-1}^{1} \sum_{\substack{j=-1 \\
(i, j) \neq(0,0)}}^{1} \tilde{w}_{i} \tilde{w}_{j} f(i h, j h)-\int_{-h}^{h} \int_{-h}^{h} f(x, y) d y d x .
\end{aligned}
$$

Recall that the integrand of $I$ is defined by

$$
f(x, y)=K(x, y) p(x, y) .
$$

To demonstrate our reasoning, we assume that $p(x, y)=p(x)=x g(x)$ and that $\eta(x, y)=0$. We comment later on the general case. Using these simplifications, we 
have

$$
f(x, y)=\frac{x g(x)}{\left(x^{2}+y^{2}\right)^{3 / 2}} .
$$

Using Taylor's theorem, we can write

$$
f(x, y)=\frac{x}{\left(x^{2}+y^{2}\right)^{3 / 2}}\left[\sum_{k=0}^{2 m} \frac{x^{k}}{k !} g^{(k)}(0)+\int_{0}^{x} \frac{(x-\tau)^{2 m}}{(2 m) !} g^{(2 m+1)}(\tau) d \tau\right] .
$$

If we define

$$
C_{k}(x, y)=\frac{g^{(k)}(0) x^{k+1}}{k !\left(x^{2}+y^{2}\right)^{3 / 2}}
$$

and

$$
R(x, y)=\int_{0}^{x} \frac{x(x-\tau)^{(2 m)}}{(2 m) !\left(x^{2}+y^{2}\right)^{3 / 2}} g^{(2 m+1)}(\tau) d \tau
$$

then

$$
f(x, y)=\sum_{k=0}^{2 m} C_{k}(x, y)+R(x, y) .
$$

Since $C_{k}$ is odd when $k$ is even, we plug $C_{k}$ into the expression for $E_{L}$ and get

$$
E_{L}\left(C_{k}\right)=0 \text { for } k \text { even. }
$$

Using this, and the fact that $E_{L}$ is linear, we can write

$$
\begin{aligned}
E_{L}(f(x, y)) & =E_{L}\left(\sum_{k=0}^{2 m} C_{k}(x, y)\right)+E_{L}(R(x, y)) \\
& =\sum_{k=1}^{m} E_{L}\left(C_{2 k-1}(x, y)\right)+E_{L}(R(x, y)) \\
& =E_{L C}+E_{L R} .
\end{aligned}
$$

Using the definition of $E_{L}$ we have

$$
\begin{aligned}
& E_{L C}=4 \sum_{k=1}^{m}\left\{-\sum_{\gamma=1}^{m} \frac{h^{2 \gamma} B_{2 \gamma}}{(2 \gamma) !}\left[\int_{0}^{h} D_{x}^{2 \gamma-1} C_{2 k-1}(h, y) d y\right.\right. \\
& +\int_{0}^{h} D_{y}^{2 \gamma-1} C_{2 k-1}(x, h) d x+\sum_{\mu=1}^{m} \frac{h^{2 \mu} B_{2 \mu}}{(2 \mu !)} D_{x}^{2 \gamma-1} D_{y}^{2 \mu-1} C_{2 k-1}(h, h) \\
& \left.+h^{2 m+1} \int_{0}^{h} \tilde{D}_{x}^{2 m+1} D_{y}^{2 \gamma-1} C_{2 k-1}(x, h) d x+h^{2 m+1} \int_{0}^{h} D_{x}^{2 \gamma-1} \tilde{D}_{y}^{2 m+1} C_{2 k-1}(h, y) d y\right] \\
& +h^{2 m+1} \int_{h}^{\pi} \int_{0}^{\pi} \tilde{D}_{x}^{2 m+1} C_{2 k-1}(x, y) d y d x+h^{2 m+1} \int_{0}^{h} \int_{h}^{\pi} \tilde{D}_{x}^{2 m+1} C_{2 k-1}(x, y) d y d x \\
& +h^{2 m+1} \int_{h}^{\pi} \int_{0}^{\pi} \tilde{D}_{y}^{2 m+1} C_{2 k-1}(x, y) d x d y+h^{2 m+1} \int_{0}^{h} \int_{h}^{\pi} \tilde{D}_{y}^{2 m+1} C_{2 k-1}(x, y) d x d y
\end{aligned}
$$




$$
\begin{aligned}
& +h^{4 m+2} \int_{h}^{\pi} \int_{0}^{\pi} \tilde{D}_{x}^{2 m+1} \tilde{D}_{y}^{2 m+1} C_{2 k-1}(x, y) d y d x \\
& +h^{4 m+2} \int_{0}^{h} \int_{h}^{\pi} \tilde{D}_{x}^{2 m+1} \tilde{D}_{y}^{2 m+1} C_{2 k-1}(x, y) d y d x \\
& \left.-\int_{0}^{h} \int_{0}^{h} C_{2 k-1}(x, y)+\frac{h^{2}}{4}\left[C_{2 k-1}(h, 0)+C_{2 k-1}(h, h)\right]\right\} .
\end{aligned}
$$

Here we have taken advantage of the symmetry of $C_{2 k-1}(x, y)$ with respect to $x$ and $y$ to reduce the domain of integration to the first quadrant. Now, let $x=h x^{\prime}$ and $y=h y^{\prime}$, substitute, and then drop the primes. The result is

$$
\begin{aligned}
& E_{L C}=\sum_{k=1}^{m} 4 h^{2 k-1}\left\{-\sum_{\gamma=1}^{m} \frac{B_{2 \gamma}}{(2 \gamma) !}\left[\int_{0}^{1} D_{x}^{2 \gamma-1} C_{2 k-1}(1, y) d y\right.\right. \\
& +\int_{0}^{1} D_{x}^{2 \gamma-1} C_{2 k-1}(x, 1) d x+\sum_{\mu=1}^{m} \frac{B_{2 \mu}}{(2 \mu !)} D_{x}^{2 \gamma-1} D_{y}^{2 \mu-1} C_{2 k-1}(1,1) \\
& \left.+\int_{0}^{1} \hat{D}_{x}^{2 m+1} D_{y}^{2 \gamma-1} C_{2 k-1}(x, 1) d x+\int_{0}^{1} D_{x}^{2 \gamma-1} \hat{D}_{y}^{2 m+1} C_{2 k-1}(1, y) d y\right] \\
& +\int_{1}^{n / 2} \int_{0}^{n / 2} \hat{D}_{x}^{2 m+1} C_{2 k-1}(x, y) d y d x+\int_{0}^{1} \int_{1}^{n / 2} \hat{D}_{x}^{2 m+1} C_{2 k-1}(x, y) d y d x \\
& +\int_{1}^{n / 2} \int_{0}^{n / 2} \hat{D}_{y}^{2 m+1} C_{2 k-1}(x, y) d x d y+\int_{0}^{1} \int_{1}^{n / 2} \hat{D}_{y}^{2 m+1} C_{2 k-1}(x, y) d x d y \\
& +\int_{1}^{n / 2} \int_{0}^{n / 2} \hat{D}_{x}^{2 m+1} \hat{D}_{y}^{2 m+1} C_{2 k-1}(x, y) d y d x \\
& +\int_{0}^{1} \int_{1}^{n / 2} \hat{D}_{x}^{2 m+1} \hat{D}_{y}^{2 m+1} C_{2 k-1}(x, y) d y d x \\
& \left.-\int_{0}^{1} \int_{0}^{1} C_{2 k-1}(x, y) d x d y+\frac{1}{4}\left[C_{2 k-1}(1,0)+C_{2 k-1}(1,1)\right]\right\}
\end{aligned}
$$

where $\hat{D}$ is defined by

$$
\hat{D}_{x}^{j} \equiv P_{j}(x) \frac{\partial^{j}}{\partial x^{j}} .
$$

We have written this as a series in odd powers of $h$. However, the coefficients are not independent of $h$ because some of the double integrals have $n / 2(=\pi / h)$ in the limits of integration. We rewrite $E_{L C}$ as follows:

$$
\begin{aligned}
& E_{L C}=\sum_{k=1}^{m} 4 h^{2 k-1}\left\{-\sum_{\gamma=1}^{m} \frac{B_{2 \gamma}}{(2 \gamma) !}\left[\int_{0}^{1} D_{x}^{2 \gamma-1} C_{2 k-1}(1, y) d y\right.\right. \\
& +\int_{0}^{1} D_{y}^{2 \gamma-1} C_{2 k-1}(x, 1) d x+\sum_{\mu=1}^{m} \frac{B_{2 \mu}}{(2 \mu !)} D_{x}^{2 \gamma-1} D_{y}^{2 \mu-1} C_{2 k-1}(1,1) \\
& \left.+\int_{0}^{1} \hat{D}_{x}^{2 m+1} D_{y}^{2 \gamma-1} C_{2 k-1}(x, 1) d x+\int_{0}^{1} D_{x}^{2 \gamma-1} \hat{D}_{y}^{2 m+1} C_{2 k-1}(1, y) d y\right]
\end{aligned}
$$




$$
\begin{aligned}
& +\int_{1}^{\infty} \int_{0}^{\infty} \hat{D}_{x}^{2 m+1} C_{2 k-1}(x, y) d y d x+\int_{0}^{1} \int_{1}^{\infty} \hat{D}_{x}^{2 m+1} C_{2 k-1}(x, y) d y d x \\
& +\int_{1}^{\infty} \int_{0}^{\infty} \hat{D}_{y}^{2 m+1} C_{2 k-1}(x, y) d x d y+\int_{0}^{1} \int_{1}^{\infty} \hat{D}_{y}^{2 m+1} C_{2 k-1}(x, y) d x d y \\
& +\int_{1}^{\infty} \int_{0}^{\infty} \hat{D}_{x}^{2 m+1} \hat{D}_{y}^{2 m+1} C_{2 k-1}(x, y) d y d x \\
& +\int_{0}^{1} \int_{1}^{\infty} \hat{D}_{x}^{2 m+1} \hat{D}_{y}^{2 m+1} C_{2 k-1}(x, y) d y d x \\
& \left.-\int_{0}^{1} \int_{0}^{1} C_{2 k-1}(x, y) d x d y+\frac{1}{4}\left[C_{2 k-1}(1,0)+C_{2 k-1}(1,1)\right]\right\}+E_{L E},
\end{aligned}
$$

where $E_{L E}$ is defined by

$$
\begin{aligned}
& E_{L E}=-\sum_{k=1}^{m} 4 h^{2 k-1}\left\{\int_{n / 2}^{\infty} \int_{0}^{\infty} \hat{D}_{x}^{2 m+1} C_{2 k-1}(x, y) d y d x\right. \\
& +4 \int_{0}^{n / 2} \int_{n / 2}^{\infty} \hat{D}_{x}^{2 m+1} C_{2 k-1}(x, y) d y d x \\
& +\int_{n / 2}^{\infty} \int_{0}^{\infty} \hat{D}_{y}^{2 m+1} C_{2 k-1}(x, y) d x d y+4 \int_{0}^{n / 2} \int_{n / 2}^{\infty} \hat{D}_{y}^{2 m+1} C_{2 k-1}(x, y) d x d y \\
& +\int_{n / 2}^{\infty} \int_{0}^{\infty} \hat{D}_{x}^{2 m+1} \hat{D}_{y}^{2 m+1} C_{2 k-1}(x, y) d y d x \\
& \left.+\int_{0}^{n / 2} \int_{n / 2}^{\infty} \hat{D}_{x}^{2 m+1} D_{y}^{2 m+1} C_{2 k-1}(x, y) d y d x\right\}
\end{aligned}
$$

Because the range of integration of the double integrals is infinite, we must discuss the issue of convergence. We examine

$$
\frac{\partial^{L}}{\partial x^{Q} y^{L-Q}} C_{2 k-1}(x, y),
$$

where $L \geq 2 m+1$ and $Q \geq 0$. This is equivalent to examining

$$
\frac{\partial^{L}}{\partial x^{Q} y^{L-Q}} \frac{x^{P}}{\left(x^{2}+y^{2}\right)^{3 / 2}}
$$

for values of $0<P \leq 2 m$. If we perform the differentiation and convert to polar coordinates, all of the terms involve $r^{P-L-3}$. Because of the restrictions on $P$ and $L$, $P-L-3<-3$. Because the radial factor has an $r$-dependence which goes as $r^{-4}$, all of the infinite integrals converge.

If we change back to the original variables (that is, let $x=x^{\prime} / h, y=y^{\prime} / h$, substitute, then drop the primes), then we have

$$
\begin{aligned}
& E_{L E}=-\sum_{k=1}^{m} 4 h^{2 m+1}\left\{\int_{\pi}^{\infty} \int_{0}^{\infty} \tilde{D}_{x}^{2 m+1} C_{2 k-1}(x, y) d y d x\right. \\
& +\int_{0}^{\pi} \int_{\pi}^{\infty} \tilde{D}_{x}^{2 m+1} C_{2 k-1}(x, y) d y d x
\end{aligned}
$$




$$
\begin{aligned}
& +\int_{\pi}^{\infty} \int_{0}^{\infty} \tilde{D}_{y}^{2 m+1} C_{2 k-1}(x, y) d x d y+\int_{0}^{\pi} \int_{\pi}^{\infty} \tilde{D}_{y}^{2 m+1} C_{2 k-1}(x, y) d x d y \\
& +h^{2 m+1} \int_{\pi}^{\infty} \int_{0}^{\infty} \tilde{D}_{x}^{2 m+1} \tilde{D}_{y}^{2 m+1} C_{2 k-1}(x, y) d y d x \\
& \left.+h^{2 m+1} \int_{0}^{\pi} \int_{\pi}^{\infty} \tilde{D}_{x}^{2 m+1} \tilde{D}_{y}^{2 m+1} C_{2 k-1}(x, y) d y d x\right\}
\end{aligned}
$$

We know that the periodic Bernoulli functions are bounded. We define $A$ as follows: $\left|P_{m}(x / h)\right| \leq A$ for all $x$. We now have a bound on $E_{L E}$ :

$$
\begin{aligned}
& E_{L E} \leq 4 A h^{2 m+1} \sum_{k=1}^{m}\left\{\int_{\pi}^{\infty} \int_{0}^{\infty}\left|D_{x}^{2 m+1} C_{2 k-1}(x, y)\right| d y d x\right. \\
& +\int_{0}^{\pi} \int_{\pi}^{\infty}\left|D_{x}^{2 m+1} C_{2 k-1}(x, y)\right| d y d x \\
& +\int_{\pi}^{\infty} \int_{0}^{\infty}\left|D_{y}^{2 m+1} C_{2 k-1}(x, y)\right| d x d y+\int_{0}^{\pi} \int_{\pi}^{\infty}\left|D_{y}^{2 m+1} C_{2 k-1}(x, y)\right| d x d y \\
& +A h^{2 m+1} \int_{\pi}^{\infty} \int_{0}^{\infty}\left|D_{x}^{2 m+1} D_{y}^{2 m+1} C_{2 k-1}(x, y)\right| d y d x \\
& \left.+A h^{2 m+1} \int_{0}^{\pi} \int_{\pi}^{\infty}\left|D_{x}^{2 m+1} D_{y}^{2 m+1} C_{2 k-1}(x, y)\right| d y d x\right\} .
\end{aligned}
$$

We have already shown that these integrals converge. Therefore, the coefficient of $h^{2 m+1}$ in $E_{L E}$ is bounded as $h$ approaches 0 . We conclude from this that $E_{L E}=$ $O\left(h^{2 m}\right)$. We now express $E_{L C}$ in the general form

$$
\begin{aligned}
E_{L C} & =\sum_{k=1}^{m} D_{2 k-1} h^{2 k-1}+E_{L E} \\
& =\sum_{k=1}^{m} D_{2 k-1} h^{2 k-1}+o\left(h^{2 m}\right),
\end{aligned}
$$

where $D_{2 k-1}$ is defined by (4.41). The analysis of $E_{L R}$ is similar and is left until later in the appendix. We show there that

$$
E_{L R}=O\left(h^{2 m-1}\right)
$$

Using (A.32), (A.49), and (A.50), we can express $E$ as follows:

$$
E=E_{\Omega}+E_{L}=E_{\Omega}+E_{L C}+E_{L R}=\sum_{k=1}^{m-1} D_{2 k-1} h^{2 k-1}+O\left(h^{2 m-1}\right) .
$$

A.5. The general case. We now briefly discuss the more general case of (A.34), where $\eta(x, y) \neq 0$ and $p$ is a function of both $x$ and $y$. In this case we have

$$
f(x, y)=\frac{p(x, y)}{\left(x^{2}+y^{2}+(\eta(0,0)-\eta(x, y))^{2}\right)^{3 / 2}} .
$$

We want to calculate and analyze $E$ as in the previous section. First, we note that the analysis of $E_{\Omega}$ does not change in this case. To analyze $E_{L}$ we rewrite $f(x, y)$ as in section 4.1. That is, we write

$$
f(x, y)=C_{0}+C_{1}+C_{2}+C_{3}+\cdots+R(x, y),
$$


where $C_{n}$ is homogeneous of order $n-2$ in $(x, y)$. We are interested in calculating

$$
E_{L}(f)=E_{L}\left(C_{0}\right)+E_{L}\left(C_{1}\right)+\cdots+E_{L}(R) .
$$

For $n$ even, $C_{n}$ is odd in $(x, y)$ so we have

$$
E_{L}\left(C_{n}\right)=0 \text { for } n \text { even. }
$$

The analysis of the nonzero terms in (A.54) is similar to the analysis of the previous section in terms of the basic method, but the details are significantly more complicated. We omit the full proof and state the result

$$
E_{L}(f)=D_{1} h+D_{3} h^{3}+\cdots+O\left(h^{N}\right),
$$

where we compute $D_{k}$ using a formula similar to (4.45). The primary difference is that the symmetry of the problem leading to (4.45) allows a simpler formula than in this more general case.

A.6. Analysis of $\boldsymbol{E}_{\boldsymbol{\Omega}}$. We have

$$
\begin{aligned}
E_{\Omega}= & h^{2 m+1} \iint_{\Omega} P_{2 m+1}(x / h) D_{x}^{2 m+1} f(x, y) d y d x \\
& +h^{2 m+1} \iint_{\Omega} P_{2 m+1}(y / h) D_{y}^{2 m+1} f(x, y) d y d x \\
& +h^{4 m+2} \iint_{\Omega} P_{2 m+1}(x / h) P_{2 m+1}(y / h) D_{x}^{2 m+1} D_{y}^{2 m+1} f(x, y) d y d x .
\end{aligned}
$$

We consider the first term of $E_{\Omega}$ :

$$
h^{2 m+1} \iint_{\Omega} P_{2 m+1}(x / h) D_{x}^{2 m+1} f(x, y) d x d y .
$$

We first define a new region $\Omega^{\prime}$ :

$$
\Omega^{\prime}=\left\{(x, y) \mid \sqrt{x^{2}+y^{2}} \geq 2 \pi\right\} .
$$

The region $\Omega^{\prime}$ is the portion of $\Omega$ which lies outside a circle of radius $2 \pi$. (This will lead to a more pleasant geometry.) Because the region $\Omega-\Omega^{\prime}$ is finite, we have

$$
h^{2 m+1} \iint_{\Omega-\Omega^{\prime}} P_{2 m+1}(x / h) D_{x}^{2 m+1} f(x, y) d x d y=O\left(h^{2 m+1}\right) .
$$

This follows because the integrand has no singularities on the domain of integration, and because $P_{2 m+1}(x / h)$ is bounded.

We now have to consider the integral over $\Omega^{\prime}$. We look first at a special case:

$$
f(x, y)=\frac{x g(x)}{\left(x^{2}+y^{2}\right)^{3 / 2}},
$$

where we have taken $g$ to be a function of $x$ alone, and $\eta=0$. We have the following:

$$
D_{x}^{2 m+1} f(x, y)=\frac{x g^{2 m+1}(x)}{\left(x^{2}+y^{2}\right)^{3 / 2}}+R(x, y),
$$


where it can be seen by examination that $R(x, y)$ consists of a collection of terms that vary as $r^{-p}$, where $p \geq 3$ and $r^{2}=x^{2}+y^{2}$. We know that $\left|P_{2 m+1}(x / h)\right| \leq C$ where $C$ is a constant, so

$$
h^{2 m+1}\left|\iint_{\Omega^{\prime}} P_{2 m+1}(x / h) R(x, y) d x d y\right| \leq h^{2 m+1} C \iint_{\Omega^{\prime}}|R(x, y)| d x d y .
$$

The integral can be seen to converge, given the way the integrand depends on the radial factor $r$. Thus, the coefficient of $h^{2 m+1}$ is bounded as $h$ approaches 0 . However, it is possible that the coefficient vanishes as $h$ approaches 0 . Specifically, the coefficient may be $o(1)$ or smaller in magnitude. Thus, we conclude that this term is $o\left(h^{2 m}\right)$. We note that this analysis serves only to place a bound on the magnitude of the term rather than to explicitly state the magnitude. (This does not affect the form of the expansion since $m$ is arbitrary. To explicitly determine the magnitude of the next term in the expansion, we must choose a larger value of $m$ in the Euler-Maclaurin formula.) We next define $q(x)=g^{2 m+1}(x)$. We write $q(x)$ as the sum of its odd and even constituents: $q(x)=q_{e}(x)+q_{o}(x)$, where $q_{e}$ is even in $x$ and $q_{o}$ is odd in $x$. Since $P_{2 m+1}(x / h)$ is odd in $x$, we can write

$$
\begin{aligned}
& h^{2 m+1} \iint_{\Omega^{\prime}} P_{2 m+1}(x / h) \frac{x q(x, y)}{\left(x^{2}+y^{2}\right)^{3 / 2}} d x d y \\
& =h^{2 m+1} \iint_{\Omega^{\prime}} P_{2 m+1}(x / h) \frac{x\left(q_{e}(x)+q_{o}(x)\right)}{\left(x^{2}+y^{2}\right)^{3 / 2}} \\
& =h^{2 m+1} \iint_{\Omega^{\prime}} P_{2 m+1}(x / h) \frac{x q_{e}(x)}{\left(x^{2}+y^{2}\right)^{3 / 2}},
\end{aligned}
$$

since

$$
h^{2 m+1} \iint_{\Omega^{\prime}} P_{2 m+1}(x / h) \frac{x q_{o}(x)}{\left(x^{2}+y^{2}\right)^{3 / 2}}=0
$$

(the integrand is odd in $x$ ).

For $m>1$, the function $P_{2 m+1}(x / h) q_{e}(x)$ is continuous for all $x$, odd in $x$, and satisfies the condition $P_{2 m+1}(0)=P_{2 m+1}(1)=0$. Thus, we express $P_{2 m+1}(x / h) q_{e}(x)$ as a Fourier (sine) series which converges absolutely and uniformly:

$$
P_{2 m+1}(x / h) q_{e}(x)=\sum_{i=1}^{\infty} A_{i} \sin (i x) .
$$

Because the series converges uniformly, we may integrate each term separately:

$$
\begin{aligned}
h^{2 m+1} \iint_{\Omega^{\prime}} P_{2 m+1}(x / h) \frac{x q_{e}(x)}{\left(x^{2}+y^{2}\right)^{3 / 2}} d x d y & =h^{2 m+1} \iint_{\Omega^{\prime}} \frac{x}{\left(x^{2}+y^{2}\right)} \sum_{i=1}^{\infty} A_{i} \sin (i x) \\
& =h^{2 m+1} \sum_{i=1}^{\infty} \iint_{\Omega^{\prime}} \frac{A_{i} x \sin (i x)}{\left(x^{2}+y^{2}\right)^{3 / 2}} d x d y .
\end{aligned}
$$

Next, we change to polar coordinates. If we define

$$
\operatorname{Si}(x)=\int_{0}^{x} \frac{\sin (x)}{x} d x
$$


then we have

$$
\begin{aligned}
& h^{2 m+1} \sum_{i=1}^{\infty} \iint_{\Omega^{\prime}} \frac{A_{i} x \sin (i x)}{\left(x^{2}+y^{2}\right)^{3 / 2}} d x d y \\
& =h^{2 m+1} \sum_{i=1}^{\infty} \int_{0}^{2 \pi} \int_{2 \pi}^{\infty} \frac{A_{i} \cos (\theta) \sin (i r \cos (\theta))}{r} d r d \theta \\
& =h^{2 m+1} \sum_{i=1}^{\infty} A_{i} \int_{0}^{2 \pi}\left[\frac{\pi}{2}|\cos (\theta)|-\operatorname{Si}(2 i \pi \cos (\theta)) \cos (\theta)\right] d \theta .
\end{aligned}
$$

It can be shown that the integrand of the latter integral is bounded by a constant which is determined by the maximum value of $\operatorname{Si}(x)$ (which occurs at $x=\pi$ ):

$$
\left|\frac{\pi}{2}\right| \cos (\theta)|-\operatorname{Si}(2 n \pi \cos (\theta)) \cos (\theta)| \leq \frac{\pi}{2}+\operatorname{Si}(\pi) .
$$

If we define

$$
C_{\max }=2 \pi\left[\frac{\pi}{2}+\operatorname{Si}(\pi)\right]
$$

then we can write

$$
\left|\iint_{\Omega^{\prime}} \frac{A_{i} x \sin (i x)}{\left(x^{2}+y^{2}\right)^{3 / 2}} d x d y\right| \leq C_{\max }\left|A_{i}\right|
$$

for all $i$. We know from the theory of Fourier series that $\sum_{i=1}^{\infty}\left|A_{i}\right|$ is a convergent series $[11,12]$. Hence, the series $C_{\max } \sum_{i=1}^{\infty}\left|A_{i}\right|$ also converges. This in turn implies that

$$
\sum_{i=1}^{\infty}\left|\iint_{\Omega^{\prime}} \frac{A_{i} x \sin (i x)}{\left(x^{2}+y^{2}\right)^{3 / 2}} d x d y\right|
$$

converges. From this we conclude that

$$
\begin{aligned}
& h^{2 m+1} \iint_{\Omega^{\prime}} P_{2 m+1}(x / h) \frac{x q_{e}(x)}{\left(x^{2}+y^{2}\right)^{3 / 2}} d x d y \\
& =h^{2 m+1} \sum_{i=1}^{\infty} \iint_{\Omega^{\prime}} \frac{A_{i} x \sin (i x)}{\left(x^{2}+y^{2}\right)^{3 / 2}} d x d y=O\left(h^{2 m+1}\right) .
\end{aligned}
$$

Now we treat the more general problem. That is, we want to show that

$$
h^{2 m+1} \iint_{\Omega^{\prime}} P_{2 m+1}(x / h) D_{x}^{2 m+1} f(x, y) d x d y=o\left(h^{2 m+1}\right),
$$

where

$$
f(x, y)=\frac{x g(x, y)}{\left(x^{2}+y^{2}+(\eta(0,0)-\eta(x, y))^{2}\right)^{3 / 2}} .
$$

We rewrite $f(x, y)$ in the following way:

$$
f(x, y)=f_{1}(x, y)+f_{2}(x, y)
$$


where

$$
\begin{aligned}
& f_{1}(x, y)=\frac{x g(x, y)}{\left(x^{2}+y^{2}\right)^{3 / 2}}, \\
& f_{2}(x, y)=\frac{x g(x, y)}{\left(x^{2}+y^{2}+(\eta(0,0)-\eta(x, y))^{2}\right)^{3 / 2}}-\frac{x g(x, y)}{\left(x^{2}+y^{2}\right)^{3 / 2}} .
\end{aligned}
$$

We can rewrite $f_{2}(x, y)$ in the following form:

$$
f_{2}(x, y)=\frac{x g(x, y)\left[A^{3 / 2}-(A+B)^{3 / 2}\right]}{A^{3 / 2}(A+B)^{3 / 2}},
$$

where

$$
\begin{aligned}
& A=x^{2}+y^{2}, \\
& B=(\eta(0,0)-\eta(x, y))^{2} .
\end{aligned}
$$

If we rationalize the numerator, we have

$$
f_{2}(x, y)=\frac{x g(x, y)\left[A^{3}-(A+B)^{3}\right]}{A^{3 / 2}(A+B)^{3 / 2}\left[A^{3 / 2}+(A+B)^{3 / 2}\right]} .
$$

Expanding the numerator gives

$$
f_{2}(x, y)=\frac{x g(x, y)\left[-3 A^{2} B-3 A B^{2}-B^{3}\right]}{A^{3 / 2}(A+B)^{3 / 2}\left[A^{3 / 2}+(A+B)^{3 / 2}\right]} .
$$

We now observe that in terms of the radial variable $r, A$ varies as $r^{2}$ and $B$ is bounded. Thus, in polar coordinates, $f_{2}$ varies as $r^{-4}$. Using this, we can write

$$
D_{x}^{2 m+1} f(x, y)=\frac{x D_{x}^{2 m+1} g(x, y)}{\left(x^{2}+y^{2}\right)^{3 / 2}}+R(x, y),
$$

where, as in the simpler case, $R(x, y)$ consists of a collection of terms varying as $r^{-p}$, $p \geq 3$ in the radial factor $r$. $\left(R(x, y)\right.$ includes all of the terms in $D_{x}^{2 m+1} f_{2}(x, y)$.)

The analysis proceeds essentially as before from here. The integral involving $R(x, y)$ is $o\left(h^{2 m}\right)$. We define $q(x, y)=D_{x}^{2 m+1} g(x, y)$. We can express $q(x, y)$ as a sum of terms

$$
q(x, y)=q_{e e}(x, y)+q_{e o}(x, y)+q_{o e}(x, y)+q_{o o}(x, y),
$$

where, for example, $q_{e o}$ is even in $x$ and odd in $y$. We can then use symmetry to write

$$
\iint_{\Omega^{\prime}} P_{2 m+1}(x / h) \frac{x q(x, y)}{\left(x^{2}+y^{2}\right)^{3 / 2}} d x d y=\iint_{\Omega^{\prime}} P_{2 m+1}(x / h) \frac{x q_{e e}(x, y)}{\left(x^{2}+y^{2}\right)^{3 / 2}} d x d y .
$$

Next, we express $P_{2 m+1}(x / h) q_{e e}(x, y)$ as a double Fourier series:

$$
\begin{aligned}
P_{2 m+1}(x / h) q_{e e}(x, y) & =\sum_{i=1}^{\infty} \sum_{j=1}^{\infty} A_{i j} \sin (i x) \cos (j y) \\
& =\frac{1}{2} \sum_{i=1}^{\infty} \sum_{j=1}^{\infty} A_{i j}[\sin (i x+j y)+\sin (i x-j y)] .
\end{aligned}
$$


From here, we use the uniform convergence of the series to enable us to interchange summation and integration. We use the fact that $\operatorname{Si}(x)$ is bounded to obtain a convergent series representation for

$$
\iint_{\Omega^{\prime}} P_{2 m+1}(x / h) \frac{x q(x, y)}{\left(x^{2}+y^{2}\right)^{3 / 2}} d x d y .
$$

As in the first case, we again conclude that

$$
h^{2 m+1} \iint_{\Omega^{\prime}} P_{2 m+1}(x / h) \frac{x q(x, y)}{\left(x^{2}+y^{2}\right)^{3 / 2}} d x d y=O\left(h^{2 m+1}\right)
$$

and hence,

$$
h^{2 m+1} \iint_{\Omega} P_{2 m+1}(x / h) \frac{x q(x, y)}{\left(x^{2}+y^{2}\right)^{3 / 2}} d x d y=O\left(h^{2 m+1}\right) .
$$

The condition that the Fourier series representations used are uniformly convergent relies on the premise that $g$ and the derivatives of $g$ are smooth.

We have considered one of the terms in $E_{\Omega}$. The analysis of the other terms is analogous. We conclude that

$$
E_{\Omega}=o\left(h^{2 m}\right) .
$$

A.7. Calculation of $\boldsymbol{E}_{\boldsymbol{L} \boldsymbol{R}}$. We now consider $E_{L R}$. In order to analyze $E_{L R}$, we need the following results, which are derived from Leibniz's rule for differentiation of an integral:

$$
\begin{aligned}
\frac{\partial^{k}}{\partial x^{k}} \int_{0}^{x} & \frac{x(x-\tau)^{m}}{m !\left(x^{2}+y^{2}\right)^{3 / 2}} f^{(m+1)}(\tau) d \tau \\
& =\int_{0}^{x} \frac{\partial^{k}}{\partial x^{k}}\left[\frac{x(x-\tau)^{m}}{m !\left(x^{2}+y^{2}\right)^{3 / 2}}\right] f^{(m+1)}(\tau) d \tau
\end{aligned}
$$

for $k<m+1$,

$$
\begin{aligned}
& \frac{\partial^{k}}{\partial x^{k}} \int_{0}^{x} \frac{x(x-\tau)^{m}}{m !\left(x^{2}+y^{2}\right)^{3 / 2}} f^{(m+1)}(\tau) d \tau \\
& =\int_{0}^{x} \frac{\partial^{m+1}}{\partial x^{m+1}}\left[\frac{x(x-\tau)^{m}}{m !\left(x^{2}+y^{2}\right)^{3 / 2}}\right] f^{(m+1)}(\tau) d \tau+\frac{x f^{(m+1)}(x)}{\left(x^{2}+y^{2}\right)^{3 / 2}}
\end{aligned}
$$

for $k=m+1$.

We can express $E_{L R}$ as follows:

$$
\begin{aligned}
& E_{L R}=-4 \sum_{\gamma=1}^{m} \frac{h^{2 \gamma} B_{2 \gamma}}{(2 \gamma) !}\left[\int_{0}^{h} D_{x}^{2 \gamma-1} R(h, y) d y+\int_{0}^{h} D_{y}^{2 \gamma-1} R(x, h) d x\right. \\
& +\sum_{\mu=1}^{m} \frac{h^{2 \mu} B_{2 \mu}}{(2 \mu) !} D_{x}^{2 \gamma-1} D_{y}^{2 \mu-1} R(h, h)+h^{2 m+1} \int_{0}^{h} \tilde{D}_{x}^{2 m+1} D_{y}^{2 \gamma-1} R(x, h) d x \\
& \left.+h^{2 m+1} \int_{0}^{h} D_{y}^{2 m+1} D_{x}^{2 \gamma-1} R(h, y) d y\right]
\end{aligned}
$$




$$
\begin{aligned}
& +4 h^{2 m+1} \int_{h}^{\pi} \int_{0}^{\pi} \tilde{D}_{x}^{2 m+1} R(x, y) d y d x+4 h^{2 m+1} \int_{0}^{h} \int_{h}^{\pi} \tilde{D}_{x}^{2 m+1} R(x, y) d y d x \\
& +4 h^{2 m+1} \int_{h}^{\pi} \int_{0}^{\pi} \tilde{D}_{y}^{2 m+1} R(x, y) d x d y+4 h^{2 m+1} \int_{0}^{h} \int_{h}^{\pi} \tilde{D}_{y}^{2 m+1} R(x, y) d x d y \\
& +4 h^{4 m+2} \int_{h}^{\pi} \int_{0}^{\pi} \tilde{D}_{x}^{2 m+1} \tilde{D}_{y}^{2 m+1} R(x, y) d y d x \\
& +4 h^{4 m+2} \int_{0}^{h} \int_{h}^{\pi} \tilde{D}_{x}^{2 m+1} \tilde{D}_{y}^{2 m+1} R(x, y) d y d x \\
& -\int_{-h}^{h} \int_{-h}^{h} R(x, y) d y d x+R(h, 0)+R(h, h),
\end{aligned}
$$

where

$$
R(x, y)=\int_{0}^{x} \frac{x(x-\tau)^{(2 m)}}{(2 m) !\left(x^{2}+y^{2}\right)^{3 / 2}} g^{2 m+1)}(\tau) d \tau .
$$

We start with the last terms in $E_{L R}$ :

$$
\begin{aligned}
R(h, 0) & =\int_{0}^{h} \frac{h(h-\tau)^{2 m}}{(2 m) ! h^{3}} g^{(2 m+1)}(\tau) d \tau \\
& =g^{2 m+1}(\xi) \int_{0}^{h} \frac{h(h-\tau)^{2 m}}{(2 m) ! h^{3}} d \tau \\
& =\frac{g^{2 m+1}(\xi)}{(2 m+1) !} h^{2 m-1} \\
& =O\left(h^{2 m-1}\right)
\end{aligned}
$$

by the mean value theorem for integrals. This assumes that the derivatives of $g(x)$ are bounded. In an analogous manner, it can be shown that $R(h, h)=O\left(h^{2 m-1}\right)$. Next we consider the integral

$$
\begin{aligned}
\int_{-h}^{h} \int_{-h}^{h} R(x, y) d x d y & =h^{2 m+1} \int_{-1}^{1} \int_{-1}^{1} d x d y \int_{0}^{x} \frac{x(x-\tau)^{2 m}}{(2 m) !\left(x^{2}+y^{2}\right)^{3 / 2}} g^{(2 m+1)}(h \tau) d \tau \\
& =h^{2 m+1} \int_{-1}^{1} \int_{-1}^{1} \frac{x^{2 m+2} g^{(2 m+1)}(\xi)}{(2 m+1) !\left(x^{2}+y^{2}\right)^{3 / 2}} d x d y \\
& =O\left(h^{2 m+1}\right)
\end{aligned}
$$

where, again, we have applied the mean value theorem of integrals. Next, we want to consider those terms in $E_{L R}$ which involve a single integral. For example, we can consider the first term in $E_{L R}$ :

$$
h^{2 \gamma} \int_{0}^{h} D_{x}^{2 \gamma-1} R(h, y) d y
$$

If we make the change of variables $x=h x^{\prime}, y=h y^{\prime}$, then (dropping the primes) we get

$$
\begin{aligned}
& h^{2 \gamma} \int_{0}^{h} D_{x}^{2 \gamma-1} R(h, y) d y \\
& =h^{2 m+1} \int_{0}^{1} \frac{\partial^{2 \gamma-1}}{\partial x^{2 \gamma-1}}\left[\int_{0}^{x} \frac{x(x-\tau)^{2 m}}{(2 m) !\left(x^{2}+y^{2}\right)^{3 / 2}} g^{(2 m+1)}(h \tau) d \tau\right]_{x=1} d y .
\end{aligned}
$$


Since $\gamma \leq m$, we can take the differential operator inside the integral (using (A.97)), perform the differentiation, and then apply the mean value theorem to verify that this integral is $O\left(h^{2 m+1}\right)$. Again, we have relied on the fact that $g(x, y)$ is bounded in all its derivatives. It is completely analogous to show that the terms

$$
h^{2 \gamma} h^{2 \mu} D_{x}^{2 \gamma-1} D_{y}^{2 \mu-1} R(h, h)
$$

are $O\left(h^{2 m+1}\right)$.

We have only to consider the terms involving the double integrals. To show the general approach, we consider the following term from $E_{L R}$ :

$$
h^{2 m+1} \int_{h}^{\pi} \int_{0}^{\pi} \tilde{D}_{x}^{2 m+1} R(x, y) d y d x .
$$

We know that

$$
\begin{aligned}
D_{x}^{2 m+1} R(x, y)= & \int_{0}^{x} \frac{\partial^{2 m+1}}{\partial x^{2 m+1}} \frac{x(x-\tau)^{2 m}}{(2 m) !\left(x^{2}+y^{2}\right)^{3 / 2}} g^{(2 m+1)}(\tau) d \tau \\
& +\frac{x g^{2 m+1}(x)}{\left(x^{2}+y^{2}\right)^{3 / 2}} .
\end{aligned}
$$

Applying the differential operator and using the mean value theorem gives a series of terms, which, when converted to polar coordinates, is of order $r^{-2}$. Thus, we can bound the integral as follows:

$$
\begin{aligned}
& h^{2 m+1}\left|\int_{h}^{\pi} \int_{0}^{\pi} \tilde{D}_{x}^{2 m+1} R(x, y) d y d x\right| \\
& \leq C h^{2 m+1} \int_{q_{1}}^{q_{2}} \int_{h s(\theta)}^{\pi s(\theta)} r^{-1} d r d \theta=O\left(h^{2 m}\right),
\end{aligned}
$$

where $C$ is some constant determined by the derivatives of $g$ and the periodic Bernoulli functions.

We have now considered all of the terms of $E_{L R}$ and conclude that

$$
E_{L R}=O\left(h^{2 m-1}\right) .
$$

Acknowledgments. The authors thank Tom Hou, John Lowengrub, and Mike Shelley for valuable discussion and suggestions.

\section{REFERENCES}

[1] J. Goodman, T. Y. Hou, And J. Lowengrub, Convergence of the point vortex method for the 2-D Euler equations, Comm. Pure Appl. Math., 43 (1990), pp. 415-430.

[2] J. S. Lowengrub, M. J. Shelley, and B. Merriman, High order and efficient methods for the vorticity formulation of the Euler equations, SIAM J. Sci. Comput., 14 (1993), pp. 11071142.

[3] T. Y. Hou And J. Lowengrub, Convergence of the point vortex method for the 3-D Euler equations, Comm. Pure Appl. Math., 43 (1990), pp. 965-981.

[4] I. NAVOT, An extension of the Euler-Maclaurin summation formula to functions with a branch singularity, J. Math. Phys., 40 (1961), pp. 271-276.

[5] A. Sidi And M. IsRAeli, Quadrature methods for periodic singular and weakly singular Fredholm integral equations, J. Sci. Comput., 3 (1988), pp. 201-231.

[6] T. Y. Hou, J. Lowengrub, AND R. KRASnY, Convergence of a point vortex method for vortex sheets, SIAM J. Numer. Anal., 28 (1991), pp. 308-320. 
[7] G. R. Baker, D. I. Meiron, and S. A. Orszag, Generalized vortex methods for free-surface flow problems, J. Fluid Mech., 123 (1982), pp. 477-501.

[8] G. R. Baker, D. I. Meiron, and S. A. Orszag, Boundary integral methods for axisymmetric and three-dimensional Rayleigh-Taylor instability problems, Phys. D, 12D (1984), pp. 1931.

[9] M. J. SHELlEY, A study of singularity formation in vortex-sheet motion by a spectrally accurate vortex method, J. Fluid Mech., 244 (1992), pp. 493-526.

[10] P. G. Saffman, Vortex Dynamics, Cambridge University Press, New York, 1992.

[11] J. W. Brown and R. V. Churchill, Fourier Series and Boundary Value Problems, McGrawHill, New York, 1993.

[12] G. P. Tolstov, Fourier Series, Prentice-Hall, Englewood Cliffs, N.J., 1962.

[13] L. F. Greengard, The Rapid Evaluation of Potential Fields in Particle Systems, MIT Press, Cambridge, MA, 1988. 